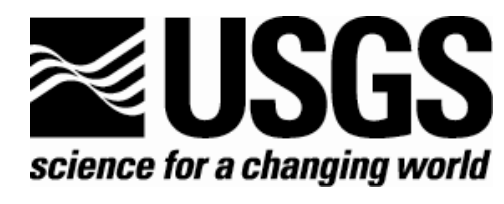

\title{
Vitrinite reflectance data for the Permian Basin, west Texas and southeast New Mexico
}

By Mark Pawlewicz, Charles E. Barker and Sargent McDonald

\section{Open-File Report 2005-1171}

U.S. Department of the Interior U.S. Geological Survey 


\title{
Vitrinite reflectance data for the Permian Basin, west Texas
}

\author{
and southeast New Mexico
}

This report presents a compilation of vitrinite reflectance $\left(\mathrm{R}_{0}\right)$ data based on analyses of samples of drill cuttings collected from 74 boreholes spread throughout the Permian Basin of west Texas and southeast New Mexico (fig. 1). The resulting data consist of 3 to 24 individual $R_{0}$ analyses representing progressively deeper stratigraphic units in each of the boreholes (table 1). The samples, Cambrian-Ordovician to Cretaceous in age, were collected at depths ranging from $200 \mathrm{ft}$ to more than 22,100 ft.

The $\mathrm{R}_{0}$ data were plotted on maps that depict three different maturation levels for organic matter in the sedimentary rocks of the Permian Basin (figs. 2-4). These maps show depths at the various borehole locations where the $\mathrm{R}_{\mathrm{o}}$ values were calculated to be 0.6 (fig. 2), 1.3 (fig. 3), and 2.0 (fig. 4) percent, which correspond, generally, to the onset of oil generation, the onset of oil cracking, and the limit of oil preservation, respectively.

The four major geologic structural features within the Permian Basin-Midland Basin, Delaware Basin, Central Basin Platform, and Northwest Shelf (fig. 1) differ in overall depth, thermal history and tectonic style. In the western Delaware Basin, for example, higher maturation is observed at relatively shallow depths, resulting from uplift and eastward basin tilting that began in the Mississippian and ultimately exposed older, thermally mature rocks. Maturity was further enhanced in this basin by the emplacement of early and mid-Tertiary intrusives. Volcanic activity also appears to have been a controlling factor for maturation of organic matter in the southern part of the otherwise tectonically stable Northwest Shelf (Barker and Pawlewicz, 1987). Depths to the three different $R_{0}$ values are greatest in the eastern Delaware Basin and southern Midland Basin. This appears to be a function of tectonic activity related to the Marathon-Ouachita orogeny, during the Late-Middle Pennsylvanian, whose affects were widespread across 
the Permian Basin. The Central Basin Platform has been a positive feature since the midto-late Paleozoic, during which time sedimentation occurred along its flanks. This nonsubsidence, along with the lack of supplemental heating (volcanism), implies lower maturation levels.

\section{Reference}

Barker, C.E., and Pawlewicz, M.J., 1987, The effects of igneous intrusions and higher heat flow on the thermal maturity of Leonardian and younger rocks, western Delaware basin, Texas, in Cromwell, D.W. ed., Glass Mountains Guidebook, Permian Basin Section, Society of Economic Paleontologists and Mineralogists, p. 69-83.

Table 1: List of boreholes in the Permian Basin, west Texas and southeast New Mexico, from which drill cuttings were collected for vitrinite reflectance analysis. Eighty-four boreholes are listed, and locations are plotted in figure 1. However, depths to the 0.6-, 1.3-, and 2.0-percent vitrinite reflectance levels were calculated for only 74 boreholes because for the other 10 , shown as green dots in figure 1 and identified here with the notation "Location not listed in figures 2-4," the stratigraphic intervals sampled were too limited for constructing meaningful regression curves to accurately determine depths.

[Map ID, borehole number (fig. 1); Lease Name, oil and gas lease name and number; Operator, borehole name; Depth ( $1^{\text {st }}$ column), sample depth in feet; $\% \mathrm{R}_{\mathrm{o}}\left(1^{\text {st }}\right.$ column $)$, mean vitrinite reflectance value; Longitude and Latitude, indicates center point within a quarter-mile grid cell in which borehole is located (proprietary agreement with database provider prevents the listing of precise longitude and latitude); Elev., surface elevation at borehole location; $\% R_{0}\left(2^{\text {nd }}\right.$ column), the three vitrinite reflectance values for which depths were calculated and plotted in figures 2-4; Depth ( $2^{\text {nd }}$ column), calculated depths corresponding to the three vitrinite reflectance values; Depth ( $3^{\text {rd }}$ column), below sea-level elevations of the three vitrinite reflectance levels.] 
Figure 1: Map of the Permian Basin, west Texas and southeast New Mexico, showing boundaries of major structural features and locations of boreholes from which samples were collected for vitrinite reflectance analysis. Numbers refer to listing of boreholes in table 1. Yellow dots indicate boreholes for which depths to the 0.6-, 1.3-, and 2.0-percent vitrinite reflectance levels were not calculated.

Figure 2: Map showing calculated depths to the 0.6-percent vitrinite reflectance value in the Permian Basin, west Texas and southeast New Mexico.

Figure 3: Map showing calculated depths to the 1.3-percent vitrinite reflectance value in the Permian Basin, west Texas and southeast New Mexico.

Figure 4: Map showing calculated depths to the 2.0-percent vitrinite reflectance value in the Permian Basin, west Texas and southeast New Mexico. 


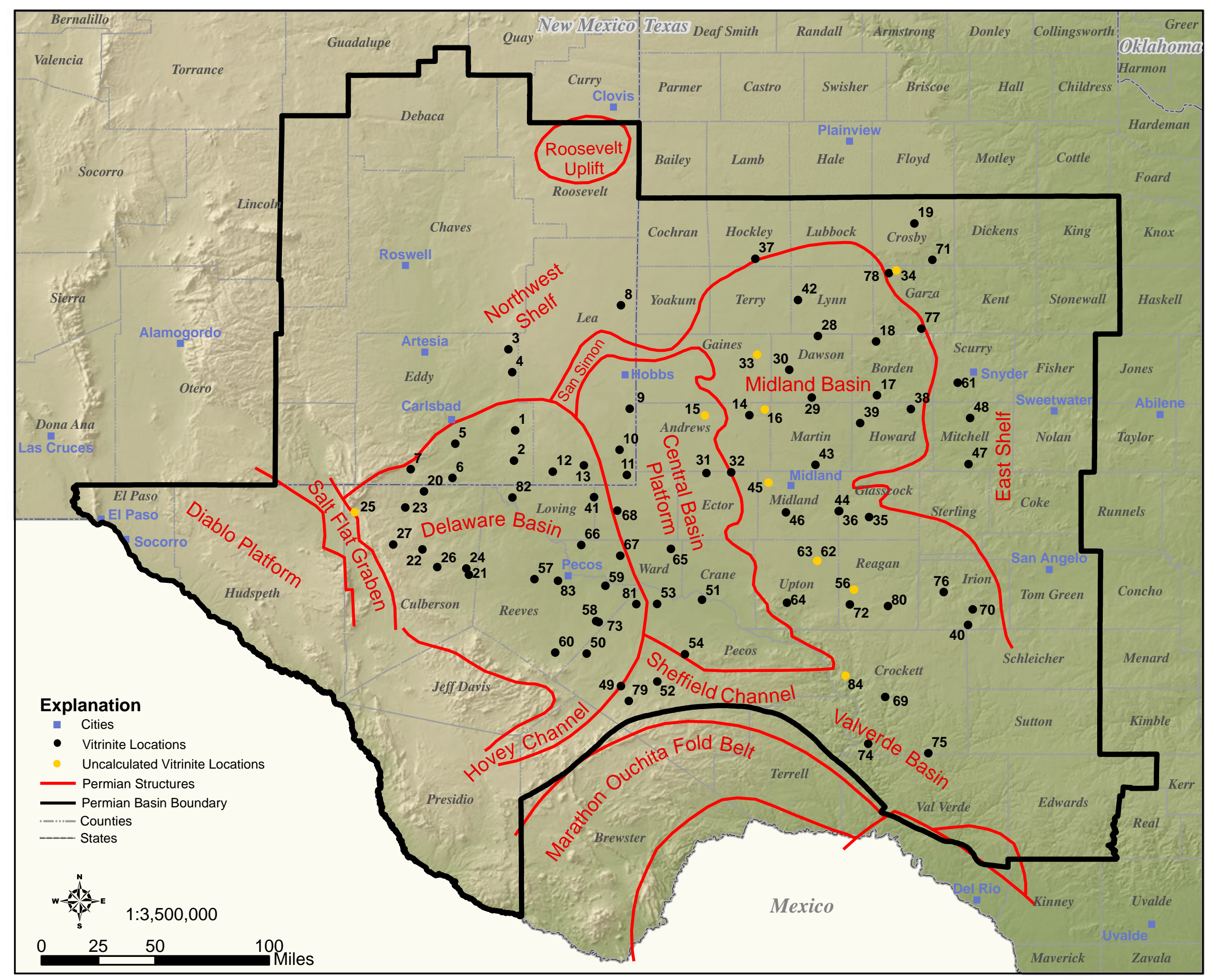

Figure 1: 


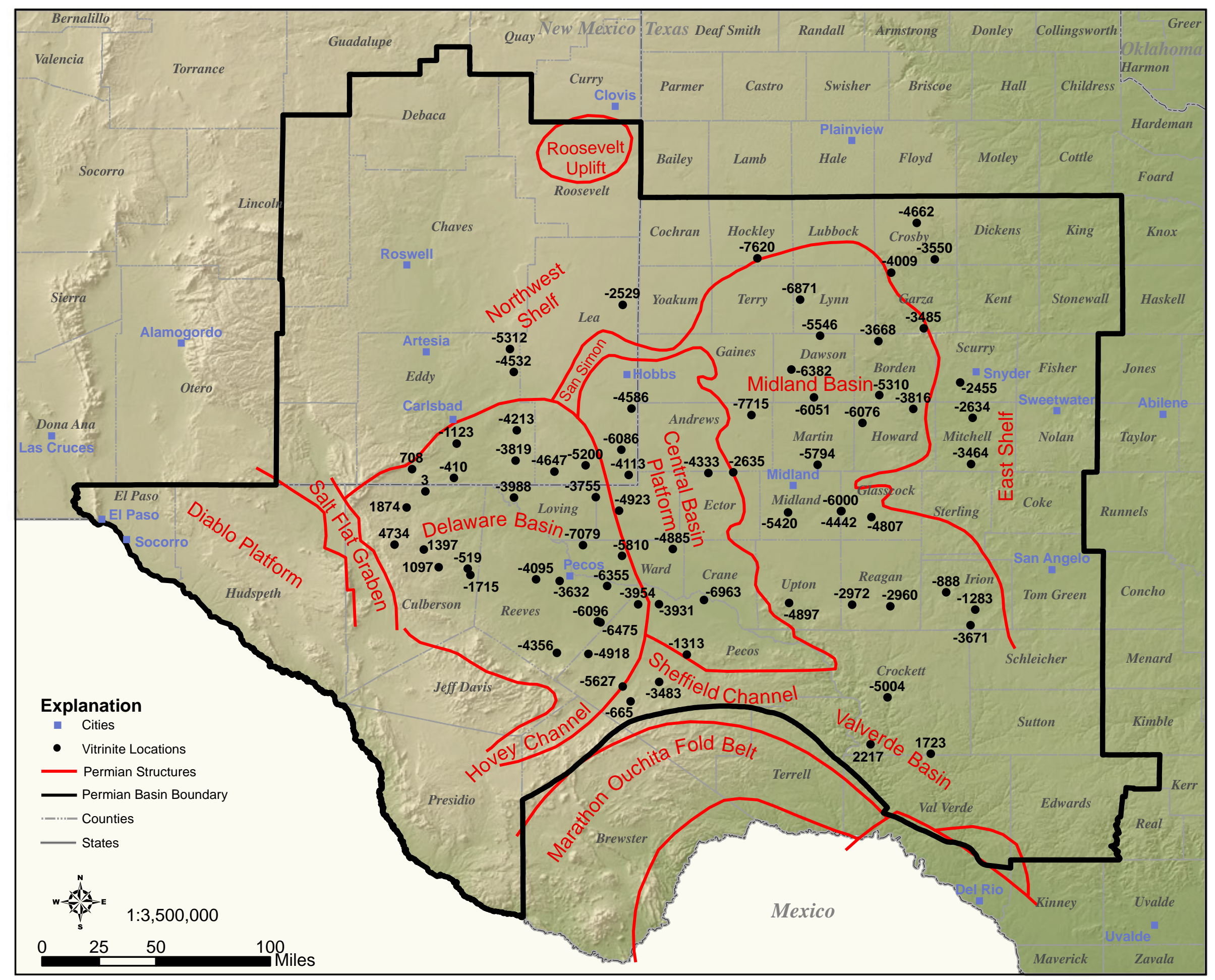

Figure 2 


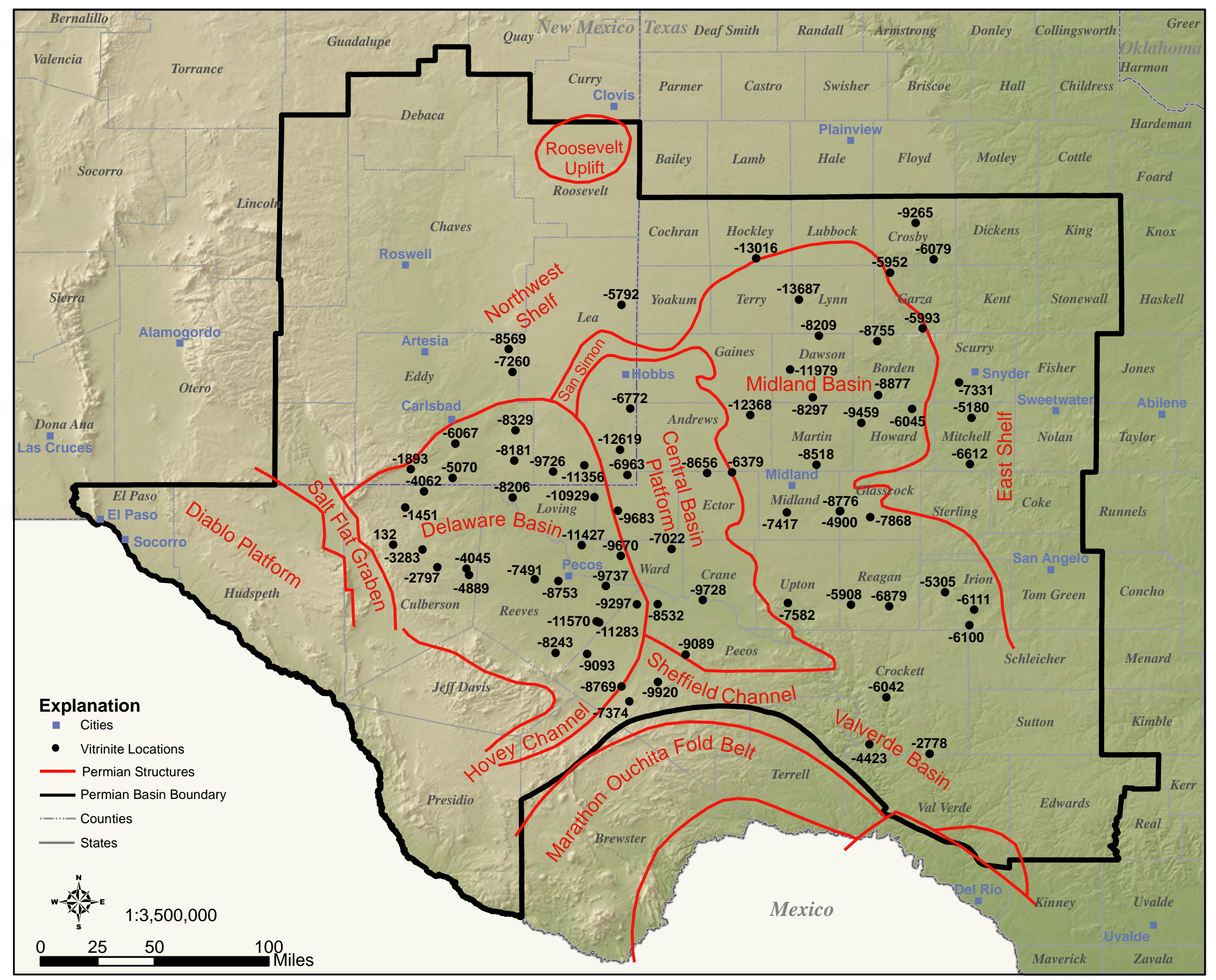

Figure 3 


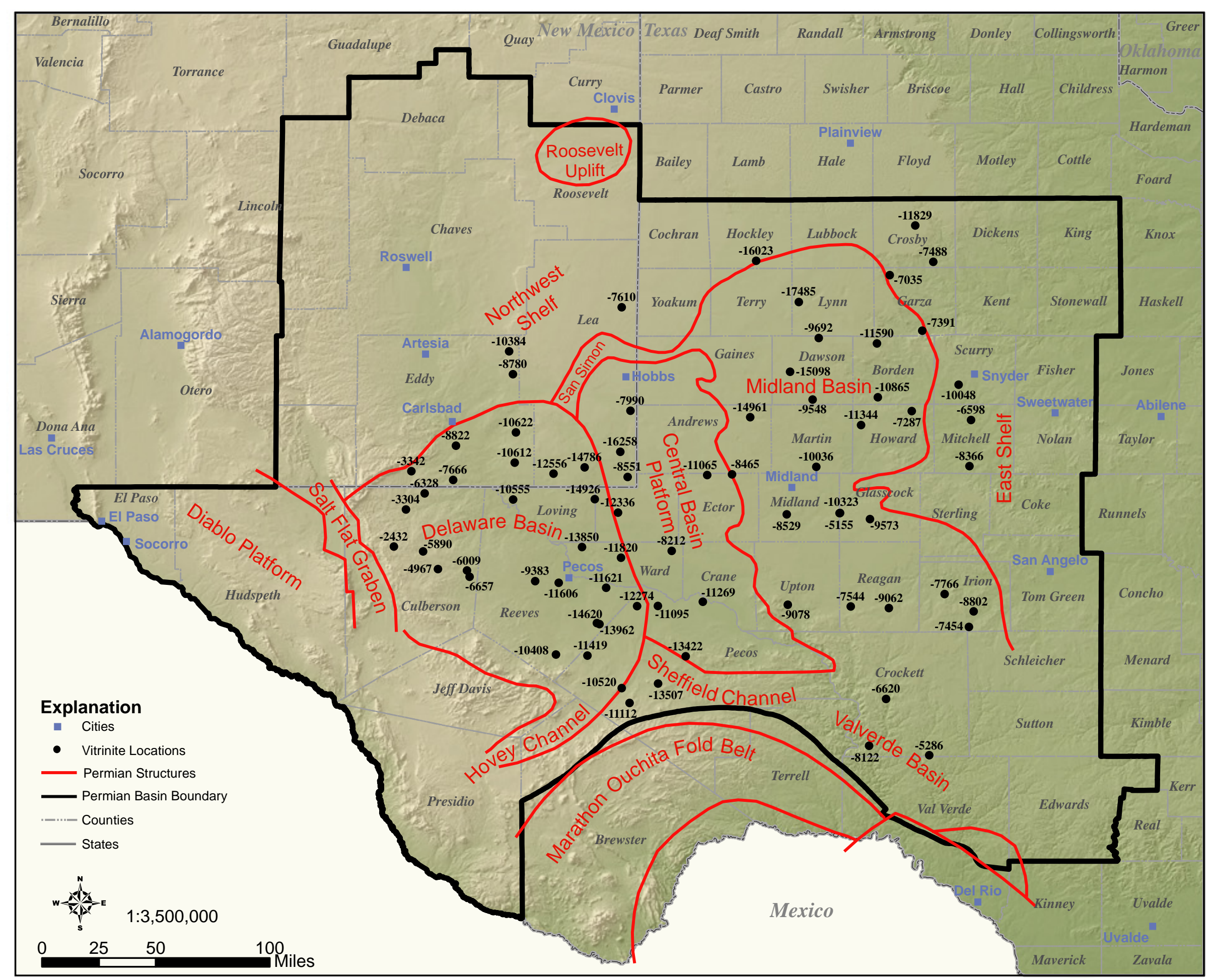

Figure 4 


\begin{tabular}{|c|c|c|c|c|c|c|c|c|c|c|}
\hline Map ID & Lease Name & Operator & Depth (ft) & \% Ro & Longitude & Latitude & Elev & \% Ro & Depth (ft) & Depth (-SL) \\
\hline 1 & J AMES RANCH UNIT 1 & SHELL OIL CO & 7990 & 0.67 & -103.83210 & 32.34022 & 3309 & 0.6 & 7522 & $-4,213$ \\
\hline 1 & J AMES RANCH UNIT I & SHELL OI L CO & 9000 & 0.75 & -103.83210 & 32.34022 & & 1.3 & 11638 & $-8,239$ \\
\hline 1 & J AMES RANCH UNIT 1 & SHELL OIL CO & 10000 & 0.87 & -103.83210 & 32.34022 & & 2 & 13931 & $-10,622$ \\
\hline 1 & J AMES RANCH UNIT 1 & SHELL OIL CO & 11000 & 1.25 & -103.83210 & 32.34022 & & & & \\
\hline 1 & J AMES RANCH UNIT 1 & SHELL OIL CO & 12130 & 1.57 & -103.83210 & 32.34022 & & & & \\
\hline 1 & J AMES RANCH UNIT 1 & SHELL OIL CO & 13040 & 1.82 & -103.83210 & 32.34022 & & & & \\
\hline 1 & J AMES RANCH UNIT 1 & SHELL OIL CO & 14050 & 2.08 & -103.83210 & 32.34022 & & & & \\
\hline 1 & J AMES RANCH UNIT 1 & SHELL OIL CO & 14990 & 2.25 & -103.83210 & 32.34022 & & & & \\
\hline 1 & J AMES RANCH UNIT 1 & SHELL OIL CO & 16990 & 3.35 & -103.83210 & 32.34022 & & & & \\
\hline 2 & J F HARRISON FEDERAL 1 & RICHARDSON \& BASS & 6990 & 0.61 & -103.83938 & 32.15142 & 3378 & 0.6 & 7197 & $-3,819$ \\
\hline 2 & J F HARRI SON FEDERAL 1 & RICHARDSON \& BASS & 8110 & 0.69 & -103.83938 & 32.15142 & & 1.3 & 11559 & $-8,181$ \\
\hline 2 & JF HARRI SON FEDERAL 1 & RICHARDSON \& BASS & 8990 & 0.81 & -103.83938 & 32.15142 & & 2 & 13990 & $-10,612$ \\
\hline 2 & JF HARRISON FEDERAL 1 & RICHARDSON \& BASS & 9490 & 0.87 & -103.83938 & 32.15142 & & & & \\
\hline 2 & J F HARRISON FEDERAL 1 & RICHARDSON \& BASS & 10530 & 1.02 & -103.83938 & 32.15142 & & & & \\
\hline 2 & J F HARRISON FEDERAL 1 & RICHARDSON \& BASS & 11590 & 1.28 & -103.83938 & 32.15142 & & & & \\
\hline 2 & JF HARRISON FEDERAL 1 & RICHARDSON \& BASS & 12485 & 1.78 & -103.83938 & 32.15142 & & & & \\
\hline 2 & JF HARRI SON FEDERAL 1 & RICHARDSON \& BASS & 13490 & 2.00 & -103.83938 & 32.15142 & & & & \\
\hline 2 & J F HARRISON FEDERAL 1 & RICHARDSON \& BASS & 14490 & 2.29 & -103.83938 & 32.15142 & & & & \\
\hline 2 & JF HARRI SON FEDERAL 1 & RICHARDSON \& BASS & 15490 & 2.45 & -103.83938 & 32.15142 & & & & \\
\hline 2 & J F HARRISON FEDERAL 1 & RICHARDSON \& BASS & 16590 & 2.57 & -103.83938 & 32.15142 & & & & \\
\hline 2 & J F HARRISON FEDERAL 1 & RICHARDSON \& BASS & 15590 & 2.57 & -103.83938 & 32.15142 & & & & \\
\hline 3 & WEST B 27 & SINCLAIR OIL \& GAS & 8490 & 0.47 & -103.87474 & 32.85839 & 3908 & 0.6 & 9220 & $-5,312$ \\
\hline 3 & WEST B 27 & SINCLAIR OIL \& GAS & 7640 & 0.47 & -103.87474 & 32.85839 & & 1.3 & 12477 & $-8,569$ \\
\hline 3 & WEST B 27 & SINCLAIR OIL \& GAS & 9460 & 0.75 & -103.87474 & 32.85839 & & 2 & 14292 & $-10,384$ \\
\hline 3 & WEST B 27 & SINCLAIR OIL \& GAS & 12105 & 0.86 & -103.87474 & 32.85839 & & & & \\
\hline 3 & WEST B 27 & SINCLAIR OIL \& GAS & 11500 & 0.87 & -103.87474 & 32.85839 & & & & \\
\hline 3 & WEST B 27 & SINCLAIR OIL \& GAS & 10500 & 1.02 & -103.87474 & 32.85839 & & & & \\
\hline 3 & WEST B 27 & SINCLAIR OIL \& GAS & 12850 & 1.48 & -103.87474 & 32.85839 & & & & \\
\hline 4 & GREENWOOD UNIT 1 & PAN AMER CORP ET AL & 7990 & 0.54 & -103.85184791 & 32.71526277 & 3657 & 0.6 & 8189 & $-4,532$ \\
\hline 4 & GREENWOOD UNIT 1 & PAN AMER CORP ET AL & 8975 & 0.81 & -103.85184791 & 32.71526277 & & 1.3 & 10917 & $-7,260$ \\
\hline 4 & GREENWOOD UNIT 1 & PAN AMER CORP ET AL & 9985 & 0.98 & -103.85184791 & 32.71526277 & & 2 & 12437 & $-8,780$ \\
\hline 4 & GREENWOOD UNIT 1 & PAN AMER CORP ET AL & 10980 & 1.41 & -103.85184791 & 32.71526277 & & & & \\
\hline 4 & GREENWOOD UNIT 1 & PAN AMER CORP ET AL & 11975 & 1.63 & -103.85184791 & 32.71526277 & & & & \\
\hline 5 & STATE LSE K-2538 1 & PHILLIPS PET ET AL & 7050 & 0.83 & -104.21207076 & 32.26250052 & 3245 & 0.6 & 4368 & $-1,123$ \\
\hline 5 & STATE LSE K-2538 1 & PHILLIPS PET ET AL & 7920 & 1.04 & -104.21207076 & 32.26250052 & & 1.3 & 9312 & $-6,067$ \\
\hline 5 & STATE LSE K-2538 1 & PHILLIPS PET ET AL & 9050 & 1.21 & -104.21207076 & 32.26250052 & & 2 & 12067 & $-8,822$ \\
\hline 5 & STATE LSE K-2538 1 & PHILLIPS PET ET AL & 10050 & 1.22 & -104.21207076 & 32.26250052 & & & & \\
\hline 5 & STATE LSE K-2538 1 & PHILLIPS PET ET AL & 6240 & 1.40 & -104.21207076 & 32.26250052 & & & & \\
\hline 5 & STATE LSE K-2538 1 & PHILLIPS PET ET AL & 11000 & 1.65 & -104.21207076 & 32.26250052 & & & & \\
\hline 5 & STATE LSE K-2538 1 & PHILLIPS PET ET AL & 12050 & 1.81 & -104.21207076 & 32.26250052 & & & & \\
\hline 5 & STATE LSE K-2538 1 & PHILLIPS PET ET AL & 13250 & 2.13 & -104.21207076 & 32.26250052 & & & & \\
\hline
\end{tabular}




\begin{tabular}{|c|c|c|c|c|c|c|c|c|c|c|}
\hline Map ID & Lease Name & Operator & Depth (ft) & \% Ro & Longitude & Latitude & Elev & \% Ro & Depth (ft) & Depth (-SL) \\
\hline 6 & OWL DRAW UNIT 1 & HAMON J AKE L & 5950 & 0.75 & -104.23254624 & 32.0436334 & 3316 & 0.6 & 3726 & -410 \\
\hline 6 & OWL DRAW UNIT 1 & HAMON J AKE L & 7050 & 0.98 & -104.23254624 & 32.0436334 & & 1.3 & 8386 & $-5,070$ \\
\hline 6 & OWL DRAW UNIT 1 & HAMON J AKE L & 7950 & 1.37 & -104.23254624 & 32.0436334 & & 2 & 10982 & $-7,666$ \\
\hline 6 & OWL DRAW UNIT 1 & HAMON J AKE L & 4350 & 1.37 & -104.23254624 & 32.0436334 & & & & \\
\hline 6 & OWL DRAW UNIT 1 & HAMON J AKE L & 8950 & 1.39 & -104.23254624 & 32.0436334 & & & & \\
\hline 6 & OWL DRAW UNIT 1 & HAMON J AKE L & 10040 & 1.79 & -104.23254624 & 32.0436334 & & & & \\
\hline 6 & OWL DRAW UNIT 1 & HAMON J AKE L & 11000 & 1.98 & -104.23254624 & 32.0436334 & & & & \\
\hline 6 & OWL DRAW UNIT 1 & HAMON J AKE L & 11990 & 2.11 & -104.23254624 & 32.0436334 & & & & \\
\hline 6 & OWL DRAW UNIT 1 & HAMON J AKE L & 13030 & 2.52 & -104.23254624 & 32.0436334 & & & & \\
\hline 7 & GOVERNMENT M 1 & CITIES SERV OIL CO & 2530 & 0.52 & -104.49594203 & 32.09651222 & 3779 & 0.6 & 3071 & 708 \\
\hline 7 & GOVERNMENT M 1 & CITIES SERV OIL CO & 3130 & 0.70 & -104.49594203 & 32.09651222 & & 1.3 & 5672 & $-1,893$ \\
\hline 7 & GOVERNMENT M 1 & CITIES SERV OIL CO & 5030 & 0.94 & -104.49594203 & 32.09651222 & & 2 & 7121 & $-3,342$ \\
\hline 7 & GOVERNMENT M 1 & CITIES SERV OIL CO & 6030 & 0.99 & -104.49594203 & 32.09651222 & & & & \\
\hline 7 & GOVERNMENT M 1 & CITIES SERV OIL CO & 4020 & 1.27 & -104.49594203 & 32.09651222 & & & & \\
\hline 7 & GOVERNMENT M 1 & CITIES SERV OI L CO & 7100 & 1.78 & -104.49594203 & 32.09651222 & & & & \\
\hline 8 & JL REED 1 & ATLANTIC REFG CO & 5030 & 0.50 & -103.16263804 & 33.13677927 & 3837 & 0.6 & 6366 & $-2,529$ \\
\hline 8 & JL REED 1 & ATLANTIC REFG CO & 6030 & 0.52 & -103.16263804 & 33.13677927 & & 1.3 & 9629 & $-5,792$ \\
\hline 8 & JL REED 1 & ATLANTIC REFG CO & 7030 & 0.70 & -103.16263804 & 33.13677927 & & 2 & 11447 & $-7,610$ \\
\hline 8 & JL REED 1 & ATLANTIC REFG CO & 8030 & 0.80 & -103.16263804 & 33.13677927 & & & & \\
\hline 8 & JL REED 1 & ATLANTIC REFG CO & 9035 & 1.12 & -103.16263804 & 33.13677927 & & & & \\
\hline 8 & J L REED 1 & ATLANTIC REFG CO & 10685 & 1.57 & -103.16263804 & 33.13677927 & & & & \\
\hline 8 & JL REED 1 & ATLANTIC REFG CO & 10230 & 1.67 & -103.16263804 & 33.13677927 & & & & \\
\hline 9 & NB BUNIN 1 & ROWAN DRILLING & 5050 & 0.40 & -103.10366534 & 32.48244355 & 3491 & 0.6 & 8077 & $-4,586$ \\
\hline 9 & NB BUNIN 1 & ROWAN DRILLING & 7050 & 0.43 & -103.10366534 & 32.48244355 & & 1.3 & 10263 & $-6,772$ \\
\hline 9 & NB BUNIN 1 & ROWAN DRI LLING & 8050 & 0.54 & -103.10366534 & 32.48244355 & & 2 & 11481 & $-7,990$ \\
\hline 9 & NB BUNIN 1 & ROWAN DRILLING & 9760 & 0.64 & -103.10366534 & 32.48244355 & & & & \\
\hline 9 & NB BUNIN 1 & ROWAN DRILLING & 8950 & 1.08 & -103.10366534 & 32.48244355 & & & & \\
\hline 10 & MEXICO 2 & SKELLY OIL COMPANY & 4070 & 0.34 & -103.16614384 & 32.21798028 & 3257 & 0.6 & 9343 & $-6,086$ \\
\hline 10 & MEXICO 2 & SKELLY OIL COMPANY & 6145 & 0.40 & -103.16614384 & 32.21798028 & & 1.3 & 15876 & $-12,619$ \\
\hline 10 & MEXICO 2 & SKELLY OIL COMPANY & 7055 & 0.48 & -103.16614384 & 32.21798028 & & 2 & 19515 & $-16,258$ \\
\hline 10 & MEXICO 2 & SKELLY OIL COMPANY & 10220 & 0.51 & -103.16614384 & 32.21798028 & & & & \\
\hline 10 & MEXICO 2 & SKELLY OIL COMPANY & 8065 & 0.53 & -103.16614384 & 32.21798028 & & & & \\
\hline 10 & MEXICO 2 & SKELLY OIL COMPANY & 9025 & 0.66 & -103.16614384 & 32.21798028 & & & & \\
\hline 11 & FEDERAL-LEONARD B 1 & HUMBLE OIL \& REFG & 7910 & 0.53 & -103.12634417 & 32.06124297 & 3015 & 0.6 & 7128 & $-4,113$ \\
\hline 11 & FEDERAL-LEONARD B 1 & HUMBLE OIL \& REFG & 6000 & 0.56 & -103.12634417 & 32.06124297 & & 1.3 & 9978 & $-6,963$ \\
\hline 11 & FEDERAL-LEONARD B 1 & HUMBLE OIL \& REFG & 8680 & 1.19 & -103.12634417 & 32.06124297 & & 2 & 11566 & $-8,551$ \\
\hline 11 & FEDERAL-LEONARD B 1 & HUMBLE OIL \& REFG & 10140 & 1.34 & -103.12634417 & 32.06124297 & & & & \\
\hline 11 & FEDERAL-LEONARD B 1 & HUMBLE OIL \& REFG & 9090 & 1.39 & -103.12634417 & 32.06124297 & & & & \\
\hline 11 & FEDERAL-LEONARD B 1 & HUMBLE OIL \& REFG & 11015 & 1.41 & -103.12634417 & 32.06124297 & & & & \\
\hline 11 & FEDERAL-LEONARD B 1 & HUMBLE OIL \& REFG & 12110 & 1.79 & -103.12634417 & 32.06124297 & & & & \\
\hline
\end{tabular}




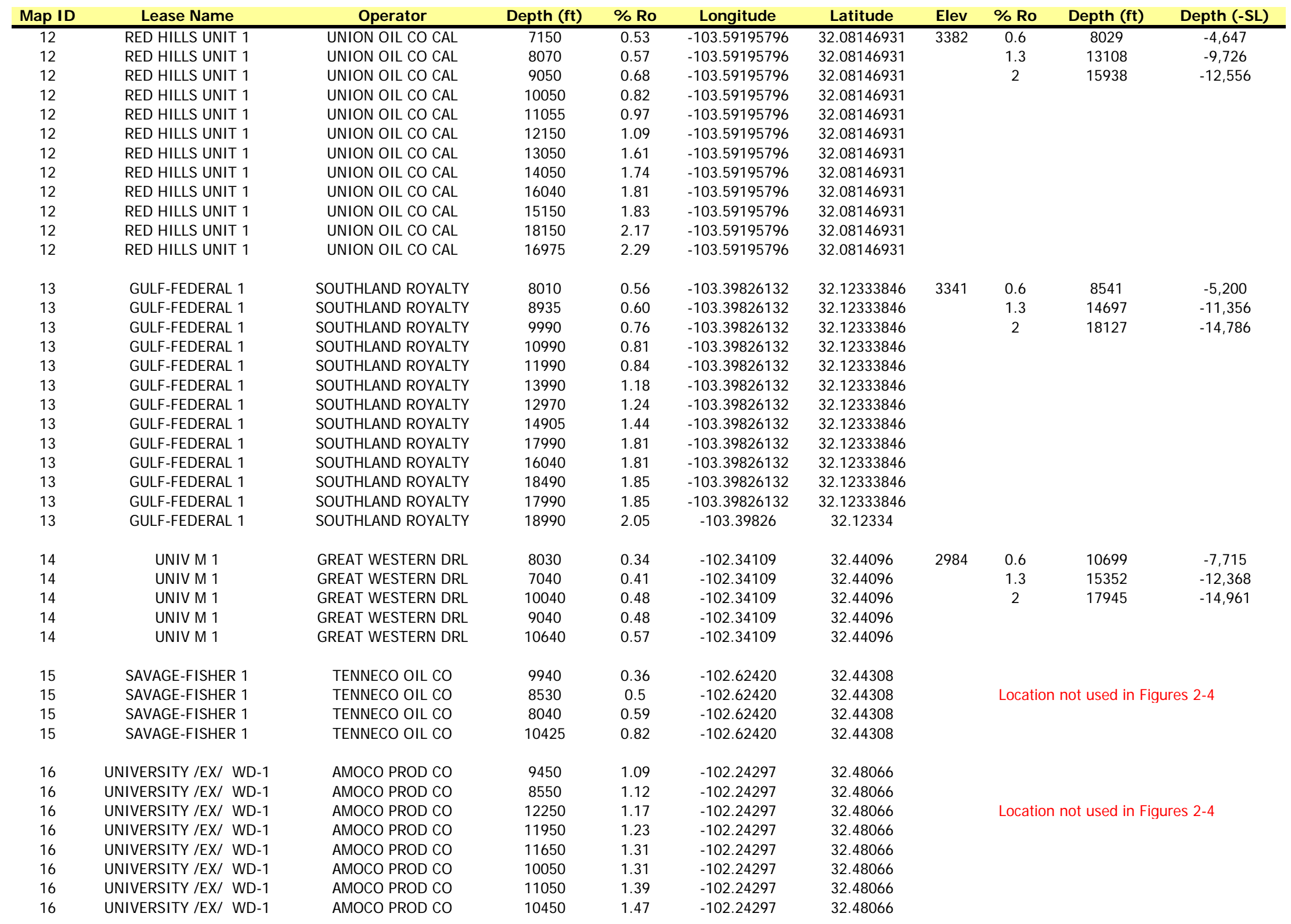




\begin{tabular}{|c|c|c|c|c|c|c|c|c|c|c|}
\hline Map ID & Lease Name & Operator & Depth (ft) & $\%$ Ro & Longitude & Latitude & Elev & $\%$ Ro & Depth (ft) & Depth (-SL) \\
\hline 17 & CLARENCE PORTER 1 & LONE STAR PROD COMP & 5980 & 0.43 & -101.53128346 & 32.57014239 & 2507 & 0.6 & 7817 & $-5,310$ \\
\hline 17 & CLARENCE PORTER 1 & LONE STAR PROD COMP & 7495 & 0.49 & -101.53128346 & 32.57014239 & & 1.3 & 11384 & $-8,877$ \\
\hline 17 & CLARENCE PORTER 1 & LONE STAR PROD COMP & 6650 & 0.49 & -101.53128346 & 32.57014239 & & 2 & 13372 & $-10,865$ \\
\hline 17 & CLARENCE PORTER 1 & LONE STAR PROD COMP & 7735 & 0.57 & -101.53128346 & 32.57014239 & & & & \\
\hline 17 & CLARENCE PORTER 1 & LONE STAR PROD COMP & 7890 & 0.62 & -101.53128346 & 32.57014239 & & & & \\
\hline 17 & CLARENCE PORTER 1 & LONE STAR PROD COMP & 8030 & 0.65 & -101.53128346 & 32.57014239 & & & & \\
\hline 18 & AM CLAYTON 1 & ANDERSN-PRICHARD OIL & 4725 & 0.50 & -101.53766419 & 32.90935146 & 2643 & 0.6 & 6311 & $-3,668$ \\
\hline 18 & AM CLAYTON 1 & ANDERSN-PRICHARD OIL & 5455 & 0.56 & -101.53766419 & 32.90935146 & & 1.3 & 11398 & $-8,755$ \\
\hline 18 & AM CLAYTON 1 & ANDERSN-PRICHARD OIL & 5830 & 0.58 & -101.53766419 & 32.90935146 & & 2 & 14233 & $-11,590$ \\
\hline 18 & AM CLAYTON 1 & ANDERSN-PRICHARD OIL & 6210 & 0.63 & -101.53766419 & 32.90935146 & & & & \\
\hline 18 & AM CLAYTON 1 & ANDERSN-PRICHARD OIL & 7360 & 0.64 & -101.53766419 & 32.90935146 & & & & \\
\hline 18 & AM CLAYTON 1 & ANDERSN-PRICHARD OIL & 7645 & 0.69 & -101.53766419 & 32.90935146 & & & & \\
\hline 18 & AM CLAYTON 1 & ANDERSN-PRICHARD OIL & 8075 & 0.71 & -101.53766419 & 32.90935146 & & & & \\
\hline 18 & AM CLAYTON 1 & ANDERSN-PRICHARD OIL & 6915 & 0.71 & -101.53766419 & 32.90935146 & & & & \\
\hline 18 & AM CLAYTON 1 & ANDERSN-PRICHARD OIL & 8325 & 0.74 & -101.53766419 & 32.90935146 & & & & \\
\hline 18 & AM CLAYTON 1 & ANDERSN-PRICHARD OIL & 8255 & 0.75 & -101.53766419 & 32.90935146 & & & & \\
\hline 18 & AM CLAYTON 1 & ANDERSN-PRICHARD OIL & 9035 & 1.03 & -101.53766419 & 32.90935146 & & & & \\
\hline 19 & NEI NDORFF MARST TR2 1 & GULF OIL CORP & 4550 & 0.35 & -101.29305631 & 33.65708846 & 3061 & 0.6 & 7723 & $-4,662$ \\
\hline 19 & NEI NDORFF MARST TR2 1 & GULF OIL CORP & 6250 & 0.36 & -101.29305631 & 33.65708846 & & 1.3 & 12326 & $-9,265$ \\
\hline 19 & NEI NDORFF MARST TR2 1 & GULF OIL CORP & 5850 & 0.37 & -101.29305631 & 33.65708846 & & 2 & 14890 & $-11,829$ \\
\hline 19 & NEI NDORFF MARST TR2 1 & GULF OIL CORP & 3530 & 0.37 & -101.29305631 & 33.65708846 & & & & \\
\hline 19 & NEI NDORFF MARST TR2 1 & GULF OIL CORP & 4050 & 0.38 & -101.29305631 & 33.65708846 & & & & \\
\hline 19 & NEINDORFF MARST TR2 1 & GULF OIL CORP & 3050 & 0.40 & -101.29305631 & 33.65708846 & & & & \\
\hline 19 & NEI NDORFF MARST TR2 1 & GULF OIL CORP & 7050 & 0.41 & -101.29305631 & 33.65708846 & & & & \\
\hline 19 & NEI NDORFF MARST TR2 1 & GULF OIL CORP & 5550 & 0.44 & -101.29305631 & 33.65708846 & & & & \\
\hline 19 & NEINDORFF MARST TR2 1 & GULF OIL CORP & 5050 & 0.44 & -101.29305631 & 33.65708846 & & & & \\
\hline 19 & NEI NDORFF MARST TR2 1 & GULF OIL CORP & 6650 & 0.48 & -101.29305631 & 33.65708846 & & & & \\
\hline 19 & NEI NDORFF MARST TR2 1 & GULF OIL CORP & 7450 & 0.53 & -101.29305631 & 33.65708846 & & & & \\
\hline 19 & NEI NDORFF MARST TR2 1 & GULF OIL CORP & 7850 & 0.54 & -101.29305631 & 33.65708846 & & & & \\
\hline 19 & NEI NDORFF MARST TR2 1 & GULF OIL CORP & 9250 & 0.61 & -101.29305631 & 33.65708846 & & & & \\
\hline 19 & NEI NDORFF MARST TR2 1 & GULF OIL CORP & 8500 & 0.70 & -101.29305631 & 33.65708846 & & & & \\
\hline 19 & NEI NDORFF MARST TR2 1 & GULF OIL CORP & 8850 & 0.71 & -101.29305631 & 33.65708846 & & & & \\
\hline 19 & NEI NDORFF MARST TR2 1 & GULF OIL CORP & 8050 & 0.72 & -101.29305631 & 33.65708846 & & & & \\
\hline 19 & NEINDORFF MARST TR2 1 & GULF OIL CORP & 8250 & 0.73 & -101.29305631 & 33.65708846 & & & & \\
\hline 20 & EE POKORNY 1 & CONTINENTAL OI L & 5030 & 0.70 & -104.41254671 & 31.95781104 & 3907 & 0.6 & 3904 & 3 \\
\hline 20 & EE POKORNY 1 & CONTI NENTAL OI L & 5280 & 0.78 & -104.41254671 & 31.95781104 & & 1.3 & 7969 & $-4,062$ \\
\hline 20 & EE POKORNY 1 & CONTI NENTAL OIL & 8030 & 1.56 & -104.41254671 & 31.95781104 & & 2 & 10235 & $-6,328$ \\
\hline 20 & EE POKORNY 1 & CONTI NENTAL OI L & 11030 & 2.24 & -104.41254671 & 31.95781104 & & & & \\
\hline 20 & EE POKORNY 1 & CONTI NENTAL OI L & 10530 & 2.30 & -104.41254671 & 31.95781104 & & & & \\
\hline 20 & EE POKORNY 1 & CONTI NENTAL OIL & 11975 & 2.38 & -104.41254671 & 31.95781104 & & & & \\
\hline 21 & GRISHAM-HUNTER-STAT 1 & EL PASO NAT GAS & 5070 & 0.59 & -104.12703311 & 31.42777335 & 3416 & 0.6 & 5131 & $-1,715$ \\
\hline
\end{tabular}




\begin{tabular}{|c|c|c|c|c|c|c|c|c|c|c|}
\hline Map ID & Lease Name & Operator & Depth (ft) & $\%$ Ro & Longitude & Latitude & Elev & $\%$ Ro & Depth (ft) & Depth (-SL) \\
\hline 21 & GRISHAM-HUNTER-STAT 1 & EL PASO NAT GAS & 7080 & 0.91 & -104.12703311 & 31.42777335 & & 1.3 & 8305 & $-4,889$ \\
\hline 21 & GRISHAM-HUNTER-STAT 1 & EL PASO NAT GAS & 7970 & 1.35 & -104.12703311 & 31.42777335 & & 2 & 10073 & 0 \\
\hline 21 & GRISHAM-HUNTER-STAT 1 & EL PASO NAT GAS & 9100 & 1.57 & -104.12703311 & 31.42777335 & & & & \\
\hline 21 & GRISHAM-HUNTER-STAT 1 & EL PASO NAT GAS & 10010 & 2.00 & -104.12703311 & 31.42777335 & & & & \\
\hline 21 & GRISHAM-HUNTER-STAT 1 & EL PASO NAT GAS & 10500 & 2.07 & -104.12703311 & 31.42777335 & & & & \\
\hline 22 & VELMA C ROUNSAVILLE 1 & HUNT W H & 2100 & 0.56 & -104.42503867 & 31.58702224 & 4157 & 0.6 & 2760 & 1,397 \\
\hline 22 & VELMA C ROUNSAVI LLE 1 & HUNT W H & 6040 & 1.02 & -104.42503867 & 31.58702224 & & 1.3 & 7440 & $-3,283$ \\
\hline 22 & VELMA C ROUNSAVI LLE 1 & HUNT W H & 5440 & 1.10 & -104.42503867 & 31.58702224 & & 2 & 10047 & $-5,890$ \\
\hline 22 & VELMA C ROUNSAVI LLE 1 & HUNT W H & 8050 & 1.20 & -104.42503867 & 31.58702224 & & & & \\
\hline 22 & VELMA C ROUNSAVILLE 1 & HUNT W H & 7100 & 1.44 & -104.42503867 & 31.58702224 & & & & \\
\hline 22 & VELMA C ROUNSAVI LLE 1 & HUNT W H & 9000 & 1.87 & -104.42503867 & 31.58702224 & & & & \\
\hline 22 & VELMA C ROUNSAVILLE 1 & HUNT W H & 10050 & 2.11 & -104.42503867 & 31.58702224 & & & & \\
\hline 22 & VELMA C ROUNSAVI LLE 1 & HUNT W H & 10900 & 2.16 & -104.42503867 & 31.58702224 & & & & \\
\hline 23 & HOMER COWDEN A 1 & MAGNOLIA PET CO & 205 & 0.57 & -104.5303189 & 31.85465742 & 3917 & 0.6 & 2043 & 1,874 \\
\hline 23 & HOMER COWDEN A 1 & MAGNOLIA PET CO & 3505 & 0.91 & -104.5303189 & 31.85465742 & & 1.3 & 5368 & $-1,451$ \\
\hline 23 & HOMER COWDEN A 1 & MAGNOLIA PET CO & 4500 & 0.92 & -104.5303189 & 31.85465742 & & 2 & 7221 & $-3,304$ \\
\hline 23 & HOMER COWDEN A 1 & MAGNOLIA PET CO & 5005 & 1.05 & -104.5303189 & 31.85465742 & & & & \\
\hline 23 & HOMER COWDEN A 1 & MAGNOLIA PET CO & 5560 & 1.10 & -104.5303189 & 31.85465742 & & & & \\
\hline 23 & HOMER COWDEN A 1 & MAGNOLIA PET CO & 6500 & 1.72 & -104.5303189 & 31.85465742 & & & & \\
\hline 23 & HOMER COWDEN A 1 & MAGNOLIA PET CO & 7365 & 1.90 & -104.5303189 & 31.85465742 & & & & \\
\hline 23 & HOMER COWDEN A 1 & MAGNOLIA PET CO & 7840 & 1.93 & -104.5303189 & 31.85465742 & & & & \\
\hline 23 & HOMER COWDEN A 1 & MAGNOLIA PET CO & 9740 & 2.77 & -104.5303189 & 31.85465742 & & & & \\
\hline 23 & HOMER COWDEN A 1 & MAGNOLIA PET CO & 8465 & 3.22 & -104.5303189 & 31.85465742 & & & & \\
\hline 23 & HOMER COWDEN A 1 & MAGNOLIA PET CO & 8718 & 3.49 & -104.5303189 & 31.85465742 & & & & \\
\hline 23 & HOMER COWDEN A 1 & MAGNOLIA PET CO & 9055 & 3.54 & -104.5303189 & 31.85465742 & & & & \\
\hline 24 & GRISHAM-HUNTER-STAT 1 & RICHARDSON \& BASS & 7950 & 1.47 & -104.14759559 & 31.46254422 & 3542 & 0.6 & 4061 & -519 \\
\hline 24 & GRISHAM-HUNTER-STAT 1 & RICHARDSON \& BASS & 9020 & 1.62 & -104.14759559 & 31.46254422 & & 1.3 & 7587 & $-4,045$ \\
\hline 24 & GRISHAM-HUNTER-STAT 1 & RICHARDSON \& BASS & 8500 & 1.65 & -104.14759559 & 31.46254422 & & 2 & 9551 & $-6,009$ \\
\hline 24 & GRISHAM-HUNTER-STAT 1 & RICHARDSON \& BASS & 9780 & 2.13 & -104.14759559 & 31.46254422 & & & & \\
\hline 25 & WILLIAMS JA 1 & UPDIKE & 2456 & 0.7 & -104.84926 & 31.82830 & & & & \\
\hline 25 & WILLIAMS JA 1 & UPDIKE & 1145 & 0.8 & -104.84926 & 31.82830 & & & & \\
\hline 25 & WILLIAMS JA 1 & UPDIKE & 787 & 0.87 & -104.84926 & 31.82830 & & \multirow{3}{*}{\multicolumn{3}{|c|}{ Location not used in Figures 2-4 }} \\
\hline 25 & WILLIAMS JA 1 & UPDIKE & 945 & 1 & -104.84926 & 31.82830 & & & & \\
\hline 25 & WILLIAMS JA 1 & UPDIKE & 2842 & 1.06 & -104.84926 & 31.82830 & & & & \\
\hline 26 & MARVIN R WEATHERBY 1 & SUN OIL COMPANY & 3030 & 0.60 & -104.32759453 & 31.47100566 & 3844 & 0.6 & 2747 & 1,097 \\
\hline 26 & MARVIN R WEATHERBY 1 & SUN OIL COMPANY & 2040 & 0.63 & -104.32759453 & 31.47100566 & & 1.3 & 6641 & $-2,797$ \\
\hline 26 & MARVIN R WEATHERBY 1 & SUN OIL COMPANY & 4040 & 0.75 & -104.32759453 & 31.47100566 & & 2 & 8811 & $-4,927$ \\
\hline 26 & MARVIN R WEATHERBY 1 & SUN OIL COMPANY & 5040 & 0.82 & -104.32759453 & 31.47100566 & & & & \\
\hline 26 & MARVIN R WEATHERBY 1 & SUN OIL COMPANY & 6040 & 1.12 & -104.32759453 & 31.47100566 & & & & \\
\hline 26 & MARVIN R WEATHERBY 1 & SUN OIL COMPANY & 7040 & 1.51 & -104.32759453 & 31.47100566 & & & & \\
\hline 26 & MARVIN R WEATHERBY 1 & SUN OIL COMPANY & 8110 & 1.84 & -104.32759453 & 31.47100566 & & & & \\
\hline
\end{tabular}




\begin{tabular}{|c|c|c|c|c|c|c|c|c|c|c|}
\hline Map ID & Lease Name & Operator & Depth (ft) & \% Ro & Longitude & Latitude & Elev & \% Ro & Depth (ft) & Depth (-SL) \\
\hline 26 & MARVIN R WEATHERBY 1 & SUN OI L COMPANY & 9300 & 2.09 & -104.32759453 & 31.47100566 & & & & \\
\hline 27 & ROUNSAVI LLE MVC 1 & STANDARD OIL TX & 1940 & 0.76 & -104.6076528 & 31.61662292 & 4933 & 0.6 & 199 & 4,734 \\
\hline 27 & ROUNSAVI LLE MVC 1 & STANDARD OIL TX & 3940 & 1.20 & -104.6076528 & 31.61662292 & & 1.3 & 4801 & 132 \\
\hline 27 & ROUNSAVILLE MVC 1 & STANDARD OIL TX & 5040 & 1.48 & -104.6076528 & 31.61662292 & & 2 & 7365 & $-2,432$ \\
\hline 27 & ROUNSAVI LLE MVC 1 & STANDARD OIL TX & 6040 & 1.62 & -104.6076528 & 31.61662292 & & & & \\
\hline 27 & ROUNSAVILLE MVC 1 & STANDARD OIL TX & 7045 & 1.75 & -104.6076528 & 31.61662292 & & & & \\
\hline 27 & ROUNSAVILLE MVC 1 & STANDARD OI L TX & 8030 & 2.17 & -104.6076528 & 31.61662292 & & & & \\
\hline 28 & MVA SMITH 1 & STANDARD OIL TX & 6950 & 0.39 & -101.9041429 & 32.94150929 & 3083 & 0.6 & 8629 & $-5,546$ \\
\hline 28 & MVA SMITH 1 & STANDARD OIL TX & 7515 & 0.52 & -101.9041429 & 32.94150929 & & 1.3 & 11292 & $-8,209$ \\
\hline 28 & MVA SMITH 1 & STANDARD OIL TX & 8065 & 0.61 & -101.9041429 & 32.94150929 & & 2 & 12775 & $-9,692$ \\
\hline 28 & MVA SMITH 1 & STANDARD OIL TX & 8800 & 0.66 & -101.9041429 & 32.94150929 & & & & \\
\hline 28 & MVA SMITH 1 & STANDARD OIL TX & 10590 & 0.67 & -101.9041429 & 32.94150929 & & & & \\
\hline 28 & MVA SMITH 1 & STANDARD OIL TX & 9145 & 0.72 & -101.9041429 & 32.94150929 & & & & \\
\hline 28 & MVA SMITH 1 & STANDARD OIL TX & 10470 & 0.73 & -101.9041429 & 32.94150929 & & & & \\
\hline 28 & MVA SMITH 1 & STANDARD OIL TX & 9365 & 0.96 & -101.9041429 & 32.94150929 & & & & \\
\hline 28 & MVA SMITH 1 & STANDARD OIL TX & 10970 & 1.24 & -101.9041429 & 32.94150929 & & & & \\
\hline 29 & TM LATHAM EST 1 & TEXACO INC & 7730 & 0.37 & -101.94404013 & 32.54924972 & 2878 & 0.6 & 8929 & $-6,051$ \\
\hline 29 & TM LATHAM EST 1 & TEXACO INC & 8590 & 0.38 & -101.94404013 & 32.54924972 & & 1.3 & 11175 & $-8,297$ \\
\hline 29 & TM LATHAM EST 1 & TEXACO INC & 8400 & 0.39 & -101.94404013 & 32.54924972 & & 2 & 12426 & $-9,548$ \\
\hline 29 & TM LATHAM EST 1 & TEXACO INC & 6615 & 0.40 & -101.94404013 & 32.54924972 & & & & \\
\hline 29 & TM LATHAM EST 1 & TEXACO INC & 6465 & 0.40 & -101.94404013 & 32.54924972 & & & & \\
\hline 29 & TM LATHAM EST 1 & TEXACO INC & 8900 & 0.42 & -101.94404013 & 32.54924972 & & & & \\
\hline 29 & TM LATHAM EST 1 & TEXACO INC & 9250 & 0.44 & -101.94404013 & 32.54924972 & & & & \\
\hline 29 & TM LATHAM EST 1 & TEXACO INC & 9095 & 0.46 & -101.94404013 & 32.54924972 & & & & \\
\hline 29 & TM LATHAM EST 1 & TEXACO INC & 7140 & 0.46 & -101.94404013 & 32.54924972 & & & & \\
\hline 29 & TM LATHAM EST 1 & TEXACO INC & 7705 & 0.48 & -101.94404013 & 32.54924972 & & & & \\
\hline 29 & TM LATHAM EST 1 & TEXACO INC & 6810 & 0.48 & -101.94404013 & 32.54924972 & & & & \\
\hline 29 & TM LATHAM EST 1 & TEXACO INC & 7965 & 0.50 & -101.94404013 & 32.54924972 & & & & \\
\hline 29 & TM LATHAM EST 1 & TEXACO INC & 9600 & 0.51 & -101.94404013 & 32.54924972 & & & & \\
\hline 29 & TM LATHAM EST 1 & TEXACO INC & 8300 & 0.54 & -101.94404013 & 32.54924972 & & & & \\
\hline 29 & TM LATHAM EST 1 & TEXACO INC & 7525 & 0.54 & -101.94404013 & 32.54924972 & & & & \\
\hline 29 & TM LATHAM EST 1 & TEXACO INC & 9800 & 0.57 & -101.94404013 & 32.54924972 & & & & \\
\hline 29 & TM LATHAM EST 1 & TEXACO INC & 10225 & 0.60 & -101.94404013 & 32.54924972 & & & & \\
\hline 29 & TM LATHAM EST 1 & TEXACO INC & 8500 & 0.60 & -101.94404013 & 32.54924972 & & & & \\
\hline 29 & TM LATHAM EST 1 & TEXACO INC & 8700 & 0.83 & -101.94404013 & 32.54924972 & & & & \\
\hline 29 & TM LATHAM EST 1 & TEXACO INC & 8860 & 0.87 & -101.94404013 & 32.54924972 & & & & \\
\hline 29 & TM LATHAM EST 1 & TEXACO INC & 11710 & 1.09 & -101.94404013 & 32.54924972 & & & & \\
\hline 29 & TM LATHAM EST 1 & TEXACO INC & 11325 & 1.15 & -101.94404013 & 32.54924972 & & & & \\
\hline 30 & J ONES FRANK B 1 & PAN AMERICAN & 7250 & 0.40 & -102.0873156 & 32.72956121 & 3020 & 0.6 & 9402 & $-6,382$ \\
\hline 30 & J ONES FRANK B 1 & PAN AMERICAN & 6750 & 0.42 & -102.0873156 & 32.72956121 & & 1.3 & 14999 & $-11,979$ \\
\hline 30 & J ONES FRANK B 1 & PAN AMERICAN & 8450 & 0.43 & -102.0873156 & 32.72956121 & & 2 & 18118 & $-15,098$ \\
\hline 30 & J ONES FRANK B 1 & PAN AMERICAN & 5250 & 0.43 & -102.0873156 & 32.72956121 & & & & \\
\hline
\end{tabular}




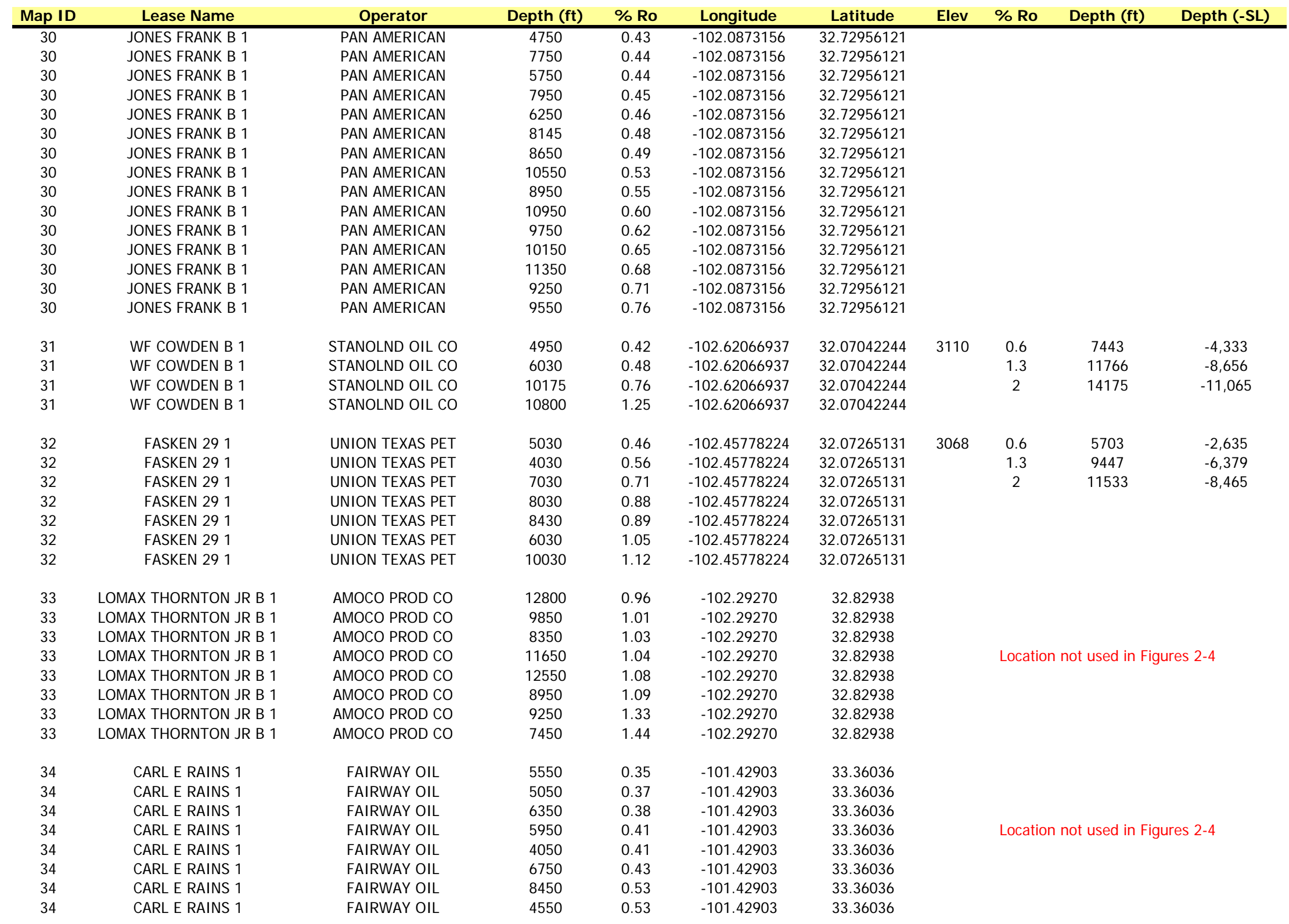




\begin{tabular}{|c|c|c|c|c|c|c|c|c|c|c|}
\hline Map ID & Lease Name & Operator & Depth (ft) & $\%$ Ro & Longitude & Latitude & Elev & \% Ro & Depth (ft) & Depth (-SL) \\
\hline 34 & CARL E RAINS 1 & FAIRWAY OIL & 8850 & 0.54 & -101.42903 & 33.36036 & & & & \\
\hline 34 & CARL E RAI NS 1 & FAI RWAY OIL & 7150 & 0.56 & -101.42903 & 33.36036 & & & & \\
\hline 34 & CARL E RAI NS 1 & FAI RWAY OIL & 3050 & 0.58 & -101.42903 & 33.36036 & & & & \\
\hline 34 & CARL E RAINS 1 & FAI RWAY OIL & 8050 & 0.62 & -101.42903 & 33.36036 & & & & \\
\hline 34 & CARL E RAI NS 1 & FAI RWAY OIL & 7530 & 0.63 & -101.42903 & 33.36036 & & & & \\
\hline 34 & CARL E RAI NS 1 & FAI RWAY OIL & 2550 & 0.71 & -101.42903 & 33.36036 & & & & \\
\hline 34 & CARL E RAI NS 1 & FAIRWAY OIL & 3550 & 0.72 & -101.42903 & 33.36036 & & & & \\
\hline 34 & CARL E RAINS 1 & FAIRWAY OIL & 7850 & 0.74 & -101.42903 & 33.36036 & & & & \\
\hline 35 & JC BRYANS 1 & MAGNOLIA PET CO & 6030 & 0.52 & -101.58039281 & 31.79404412 & 2718 & 0.6 & 7525 & $-4,087$ \\
\hline 35 & JC BRYANS 1 & MAGNOLIA PET CO & 8050 & 0.63 & -101.58039281 & 31.79404412 & & 1.3 & 10586 & $-7,868$ \\
\hline 35 & JC BRYANS 1 & MAGNOLIA PET CO & 9040 & 0.69 & -101.58039281 & 31.79404412 & & 2 & 12291 & $-9,573$ \\
\hline 35 & JC BRYANS 1 & MAGNOLIA PET CO & 10240 & 1.29 & -101.58039281 & 31.79404412 & & & & \\
\hline 35 & JC BRYANS 1 & MAGNOLIA PET CO & 10800 & 1.39 & -101.58039281 & 31.79404412 & & & & \\
\hline 36 & WA HUTCHI NSON 1 & SUN OIL COMPANY & 6883 & 0.43 & -101.77087424 & 31.82782019 & 2628 & 0.6 & 7070 & $-4,442$ \\
\hline 36 & WA HUTCHI NSON 1 & SUN OIL COMPANY & 6903 & 0.46 & -101.77087424 & 31.82782019 & & 1.3 & 7528 & $-4,900$ \\
\hline 36 & WA HUTCHI NSON 1 & SUN OIL COMPANY & 6924 & 0.48 & -101.77087424 & 31.82782019 & & 2 & 7783 & $-5,155$ \\
\hline 36 & WA HUTCHINSON 1 & SUN OIL COMPANY & 6918 & 0.48 & -101.77087424 & 31.82782019 & & & & \\
\hline 36 & WA HUTCHINSON 1 & SUN OIL COMPANY & 6984 & 0.49 & -101.77087424 & 31.82782019 & & & & \\
\hline 37 & ARMSTRONG 1 & FRENCH L R J R & 8300 & 0.42 & -102.30767267 & 33.43269235 & 3460 & 0.6 & 11080 & $-7,620$ \\
\hline 37 & ARMSTRONG 1 & FRENCH L R J R & 9000 & 0.47 & -102.30767267 & 33.43269235 & & 1.3 & 16476 & $-13,016$ \\
\hline 37 & ARMSTRONG 1 & FRENCH L R J R & 10040 & 0.48 & -102.30767267 & 33.43269235 & & 2 & 19483 & $-16,023$ \\
\hline 37 & ARMSTRONG 1 & FRENCH L R J R & 10540 & 0.58 & -102.30767267 & 33.43269235 & & & & \\
\hline 37 & ARMSTRONG 1 & FRENCH L R J R & 12040 & 0.64 & -102.30767267 & 33.43269235 & & & & \\
\hline 37 & ARMSTRONG 1 & FRENCH L R J R & 13040 & 0.80 & -102.30767267 & 33.43269235 & & & & \\
\hline 37 & ARMSTRONG 1 & FRENCH L R J R & 13490 & 0.88 & -102.30767267 & 33.43269235 & & & & \\
\hline 38 & REED NH 1 & FOREST OIL CORP & 4030 & 0.26 & -101.31807838 & 32.47918742 & 2514 & 0.6 & 6330 & $-3,816$ \\
\hline 38 & REED NH 1 & FOREST OIL CORP & 4030 & 0.26 & -101.31807838 & 32.47918742 & & 1.3 & 8559 & $-6,045$ \\
\hline 38 & REED NH 1 & FOREST OIL CORP & 5030 & 0.49 & -101.31807838 & 32.47918742 & & 2 & 9801 & $-7,287$ \\
\hline 38 & REED NH 1 & FOREST OIL CORP & 6030 & 0.50 & -101.31807838 & 32.47918742 & & & & \\
\hline 38 & REED NH 1 & FOREST OIL CORP & 6970 & 0.64 & -101.31807838 & 32.47918742 & & & & \\
\hline 38 & REED NH 1 & FOREST OIL CORP & 7870 & 1.05 & -101.31807838 & 32.47918742 & & & & \\
\hline 39 & PAGE LOLA A 1 & ANADARKO PROD & 6040 & 0.39 & -101.63943042 & 32.39135799 & 2586 & 0.6 & 8662 & $-6,076$ \\
\hline 39 & PAGE LOLA A 1 & ANADARKO PROD & 8030 & 0.44 & -101.63943042 & 32.39135799 & & 1.3 & 12045 & $-9,459$ \\
\hline 39 & PAGE LOLA A 1 & ANADARKO PROD & 9030 & 0.52 & -101.63943042 & 32.39135799 & & 2 & 13930 & $-11,344$ \\
\hline 39 & PAGE LOLA A 1 & ANADARKO PROD & 10030 & 0.97 & -101.63943042 & 32.39135799 & & & & \\
\hline 39 & PAGE LOLA A 1 & ANADARKO PROD & 10455 & 1.09 & -101.63943042 & 32.39135799 & & & & \\
\hline 39 & PAGE LOLA A 1 & ANADARKO PROD & 16010 & 2.20 & -101.63943042 & 32.39135799 & & & & \\
\hline 40 & NOELKE MONTE 1B & HANKAMER CURTIS & 5525 & 0.48 & -100.95690944 & 31.10681518 & 2430 & 0.6 & 6101 & $-3,671$ \\
\hline 40 & NOELKE MONTE 1B & HANKAMER CURTIS & 4530 & 0.49 & -100.95690944 & 31.10681518 & & 1.3 & 8530 & $-6,100$ \\
\hline 40 & NOELKE MONTE 1B & HANKAMER CURTIS & 6525 & 0.52 & -100.95690944 & 31.10681518 & & 2 & 9884 & $-7,454$ \\
\hline
\end{tabular}




\begin{tabular}{|c|c|c|c|c|c|c|c|c|c|c|}
\hline Map ID & Lease Name & Operator & Depth (ft) & $\%$ Ro & Longitude & Latitude & Elev & $\%$ Ro & Depth (ft) & Depth (-SL) \\
\hline 40 & NOELKE MONTE 1B & HANKAMER CURTIS & 7825 & 1.06 & -100.95690944 & 31.10681518 & & & & \\
\hline 41 & LINEBERRY EVELYN 1 & RODEN OI L CO & 12040 & 1.07 & -103.3278672 & 31.91793159 & 3000 & 0.6 & 6755 & $-3,755$ \\
\hline 41 & LINEBERRY EVELYN 1 & RODEN OIL CO & 13740 & 1.11 & -103.3278672 & 31.91793159 & & 1.3 & 13929 & $-10,929$ \\
\hline 41 & LI NEBERRY EVELYN 1 & RODEN OIL CO & 13030 & 1.26 & -103.3278672 & 31.91793159 & & 2 & 17926 & $-14,926$ \\
\hline 41 & LINEBERRY EVELYN 1 & RODEN OIL CO & 15040 & 1.47 & -103.3278672 & 31.91793159 & & & & \\
\hline 41 & LINEBERRY EVELYN 1 & RODEN OIL CO & 16400 & 1.90 & -103.3278672 & 31.91793159 & & & & \\
\hline 41 & LI NEBERRY EVELYN 1 & RODEN OIL CO & 18030 & 2.09 & -103.3278672 & 31.91793159 & & & & \\
\hline 41 & LINEBERRY EVELYN 1 & RODEN OIL CO & 19030 & 2.12 & -103.3278672 & 31.91793159 & & & & \\
\hline 41 & LINEBERRY EVELYN 1 & RODEN OIL CO & 17040 & 2.17 & -103.3278672 & 31.91793159 & & & & \\
\hline 41 & LINEBERRY EVELYN 1 & RODEN OI L CO & 22130 & 2.57 & -103.3278672 & 31.91793159 & & & & \\
\hline 42 & CODY BRAGG 1 & LONE STAR PROD & 4740 & 0.36 & -102.03458 & 33.17302 & 3202 & 0.6 & 10073 & $-6,871$ \\
\hline 42 & CODY BRAGG 1 & LONE STAR PROD & 5150 & 0.38 & -102.03458 & 33.17302 & & 1.3 & 16889 & $-13,687$ \\
\hline 42 & CODY BRAGG 1 & LONE STAR PROD & 6770 & 0.40 & -102.03458 & 33.17302 & & 2 & 20687 & $-17,485$ \\
\hline 42 & CODY BRAGG 1 & LONE STAR PROD & 7150 & 0.41 & -102.03458 & 33.17302 & & & & \\
\hline 42 & CODY BRAGG 1 & LONE STAR PROD & 8650 & 0.42 & -102.03458 & 33.17302 & & & & \\
\hline 42 & CODY BRAGG 1 & LONE STAR PROD & 8250 & 0.42 & -102.03458 & 33.17302 & & & & \\
\hline 42 & CODY BRAGG 1 & LONE STAR PROD & 7850 & 0.42 & -102.03458 & 33.17302 & & & & \\
\hline 42 & CODY BRAGG 1 & LONE STAR PROD & 6350 & 0.42 & -102.03458 & 33.17302 & & & & \\
\hline 42 & CODY BRAGG 1 & LONE STAR PROD & 5570 & 0.43 & -102.03458 & 33.17302 & & & & \\
\hline 42 & CODY BRAGG 1 & LONE STAR PROD & 5950 & 0.44 & -102.03458 & 33.17302 & & & & \\
\hline 42 & CODY BRAGG 1 & LONE STAR PROD & 9050 & 0.47 & -102.03458 & 33.17302 & & & & \\
\hline 42 & CODY BRAGG 1 & LONE STAR PROD & 7450 & 0.48 & -102.03458 & 33.17302 & & & & \\
\hline 42 & CODY BRAGG 1 & LONE STAR PROD & 10350 & 0.58 & -102.03458 & 33.17302 & & & & \\
\hline 42 & CODY BRAGG 1 & LONE STAR PROD & 10950 & 0.59 & -102.03458 & 33.17302 & & & & \\
\hline 42 & CODY BRAGG 1 & LONE STAR PROD & 9850 & 0.60 & -102.03458 & 33.17302 & & & & \\
\hline 42 & CODY BRAGG 1 & LONE STAR PROD & 10550 & 0.62 & -102.03458 & 33.17302 & & & & \\
\hline 42 & CODY BRAGG 1 & LONE STAR PROD & 9450 & 0.66 & -102.03458 & 33.17302 & & & & \\
\hline 43 & JW BLOCKER ETAL 1 & HUMBLE OIL \& REFG & 6445 & 0.36 & -101.92099 & 32.12386 & 2720 & 0.6 & 8514 & $-5,794$ \\
\hline 43 & JW BLOCKER ETAL 1 & HUMBLE OIL \& REFG & 7100 & 0.43 & -101.92099 & 32.12386 & & 1.3 & 11238 & $-8,518$ \\
\hline 43 & J W BLOCKER ETAL 1 & HUMBLE OIL \& REFG & 8000 & 0.47 & -101.92099 & 32.12386 & & 2 & 12756 & $-10,036$ \\
\hline 43 & JW BLOCKER ETAL 1 & HUMBLE OIL \& REFG & 9000 & 0.61 & -101.92099 & 32.12386 & & & & \\
\hline 43 & JW BLOCKER ETAL 1 & HUMBLE OIL \& REFG & 10060 & 1.16 & -101.92099 & 32.12386 & & & & \\
\hline 43 & JW BLOCKER ETAL 1 & HUMBLE OIL \& REFG & 11100 & 1.22 & -101.92099 & 32.12386 & & & & \\
\hline 43 & JW BLOCKER ETAL 1 & HUMBLE OIL \& REFG & 11500 & 1.24 & -101.92099 & 32.12386 & & & & \\
\hline 44 & MRS WA HUTCHINSON 7 & SUN OIL COMPANY & 7213 & 0.40 & -101.77939 & 31.82738 & 2628 & 0.6 & 8628 & $-6,000$ \\
\hline 44 & MRS WA HUTCHINSON 7 & SUN OIL COMPANY & 7209 & 0.41 & -101.77939 & 31.82738 & & 1.3 & 11404 & $-8,776$ \\
\hline 44 & MRS WA HUTCHINSON 7 & SUN OIL COMPANY & 7487 & 0.43 & -101.77939 & 31.82738 & & 2 & 12951 & $-10,323$ \\
\hline 44 & MRS WA HUTCHINSON 7 & SUN OIL COMPANY & 7435 & 0.43 & -101.77939 & 31.82738 & & & & \\
\hline 44 & MRS WA HUTCHINSON 7 & SUN OIL COMPANY & 7465 & 0.44 & -101.77939 & 31.82738 & & & & \\
\hline 44 & MRS WA HUTCHINSON 7 & SUN OIL COMPANY & 7787 & 0.46 & -101.77939 & 31.82738 & & & & \\
\hline 44 & MRS WA HUTCHINSON 7 & SUN OIL COMPANY & 7758 & 0.46 & -101.77939 & 31.82738 & & & & \\
\hline 44 & MRS WA HUTCHINSON 7 & SUN OIL COMPANY & 7593 & 0.46 & -101.77939 & 31.82738 & & & & \\
\hline
\end{tabular}




\begin{tabular}{|c|c|c|c|c|c|c|c|c|c|c|}
\hline Map ID & Lease Name & Operator & Depth (ft) & $\%$ Ro & Longitude & Latitude & Elev & $\%$ Ro & Depth (ft) & Depth (-SL) \\
\hline 44 & MRS WA HUTCHI NSON 7 & SUN OIL COMPANY & 7522 & 0.46 & -101.77939 & 31.82738 & & & & \\
\hline 44 & MRS WA HUTCHINSON 7 & SUN OIL COMPANY & 7863 & 0.48 & -101.77939 & 31.82738 & & & & \\
\hline 45 & DAVID FASKEN AY 1 & PAN AMERICAN & 7530 & 0.37 & -102.21999 & 32.01735 & & & & \\
\hline 45 & DAVID FASKEN AY 1 & PAN AMERICAN & 6040 & 0.47 & -102.21999 & 32.01735 & & \multicolumn{3}{|c|}{ Location not used in Figures 2-4 } \\
\hline 45 & DAVID FASKEN AY 1 & PAN AMERICAN & 7040 & 0.49 & -102.21999 & 32.01735 & & & & \\
\hline 45 & DAVID FASKEN AY 1 & PAN AMERICAN & 6530 & 0.49 & -102.21999 & 32.01735 & & & & \\
\hline 46 & BRAUN ETAL 1 & BROWN TOM INC & 7040 & 0.43 & -102.11271931 & 31.82418558 & 2870 & 0.6 & 8290 & $-5,420$ \\
\hline 46 & BRAUN ETAL 1 & BROWN TOM INC & 8040 & 0.46 & -102.11271931 & 31.82418558 & & 1.3 & 10287 & $-7,417$ \\
\hline 46 & BRAUN ETAL 1 & BROWN TOM INC & 10040 & 0.98 & -102.11271931 & 31.82418558 & & 2 & 11399 & $-8,529$ \\
\hline 46 & BRAUN ETAL 1 & BROWN TOM INC & 9040 & 1.11 & -102.11271931 & 31.82418558 & & & & \\
\hline 46 & BRAUN ETAL 1 & BROWN TOM INC & 11130 & 1.42 & -102.11271931 & 31.82418558 & & & & \\
\hline 47 & ELLWOOD EST 1 & HUMBLE OIL \& REFG & 5540 & 0.44 & -100.95144353 & 32.12725898 & 2235 & 0.6 & 5699 & $-3,464$ \\
\hline 47 & ELLWOOD EST 1 & HUMBLE OIL \& REFG & 4520 & 0.45 & -100.95144353 & 32.12725898 & & 1.3 & 8847 & $-6,612$ \\
\hline 47 & ELLWOOD EST 1 & HUMBLE OIL \& REFG & 3650 & 0.49 & -100.95144353 & 32.12725898 & & 2 & 10601 & $-8,366$ \\
\hline 47 & ELLWOOD EST 1 & HUMBLE OIL \& REFG & 6540 & 0.85 & -100.95144353 & 32.12725898 & & & & \\
\hline 47 & ELLWOOD EST 1 & HUMBLE OIL \& REFG & 7994 & 0.91 & -100.95144353 & 32.12725898 & & & & \\
\hline 47 & ELLWOOD EST 1 & HUMBLE OIL \& REFG & 7500 & 0.91 & -100.95144353 & 32.12725898 & & & & \\
\hline 48 & PRUITT KEEL 1 & HUMBLE OIL \& REFG & 4530 & 0.45 & -100.94404138 & 32.42388554 & 2156 & 0.6 & 4790 & $-2,634$ \\
\hline 48 & PRUITT KEEL 1 & HUMBLE OIL \& REFG & 3465 & 0.49 & -100.94404138 & 32.42388554 & & 1.3 & 7336 & $-5,180$ \\
\hline 48 & PRUITT KEEL 1 & HUMBLE OIL \& REFG & 5550 & 0.83 & -100.94404138 & 32.42388554 & & 2 & 8754 & $-6,598$ \\
\hline 48 & PRUITT KEEL 1 & HUMBLE OIL \& REFG & 7005 & 1.08 & -100.94404138 & 32.42388554 & & & & \\
\hline 49 & CB HARRISON ETAL 1 & ADKISSON A W & 5380 & 0.35 & -103.16077988 & 30.71791872 & 2481 & 0.6 & 8108 & $-5,627$ \\
\hline 49 & CB HARRISON ETAL 1 & ADKISSON A W & 7080 & 0.38 & -103.16077988 & 30.71791872 & & 1.3 & 11250 & $-8,769$ \\
\hline 49 & CB HARRISON ETAL 1 & ADKISSON A W & 8200 & 0.72 & -103.16077988 & 30.71791872 & & 2 & 13001 & $-10,520$ \\
\hline 49 & CB HARRISON ETAL 1 & ADKISSON A W & 9750 & 0.86 & -103.16077988 & 30.71791872 & & & & \\
\hline 49 & CB HARRISON ETAL 1 & ADKISSON A W & 11580 & 1.35 & -103.16077988 & 30.71791872 & & & & \\
\hline 50 & KELLY STATE GAS UNI 1 & ATLANTIC RICHFLD & 7020 & 0.46 & -103.38185801 & 30.92111339 & 3216 & 0.6 & 8134 & $-4,918$ \\
\hline 50 & KELLY STATE GAS UNI 1 & ATLANTIC RICHFLD & 8030 & 0.52 & -103.38185801 & 30.92111339 & & 1.3 & 12309 & $-9,093$ \\
\hline 50 & KELLY STATE GAS UNI 1 & ATLANTIC RICHFLD & 6035 & 0.60 & -103.38185801 & 30.92111339 & & 2 & 14635 & $-11,419$ \\
\hline 50 & KELLY STATE GAS UNI 1 & ATLANTIC RI CHFLD & 9020 & 0.69 & -103.38185801 & 30.92111339 & & & & \\
\hline 50 & KELLY STATE GAS UNI 1 & ATLANTIC RICHFLD & 10020 & 0.85 & -103.38185801 & 30.92111339 & & & & \\
\hline 50 & KELLY STATE GAS UNI 1 & ATLANTIC RICHFLD & 11030 & 1.21 & -103.38185801 & 30.92111339 & & & & \\
\hline 50 & KELLY STATE GAS UNI 1 & ATLANTIC RICHFLD & 12020 & 1.26 & -103.38185801 & 30.92111339 & & & & \\
\hline 50 & KELLY STATE GAS UNI 1 & ATLANTIC RICHFLD & 13020 & 1.53 & -103.38185801 & 30.92111339 & & & & \\
\hline 50 & KELLY STATE GAS UNI 1 & ATLANTIC RICHFLD & 14030 & 1.79 & -103.38185801 & 30.92111339 & & & & \\
\hline 50 & KELLY STATE GAS UNI 1 & ATLANTIC RICHFLD & 14970 & 1.96 & -103.38185801 & 30.92111339 & & & & \\
\hline 51 & WA WRIGHT 1 & STANOLND OIL CO & 11510 & 0.56 & -102.64819034 & 31.26486416 & 2391 & 0.6 & 9354 & $-6,963$ \\
\hline 51 & WA WRIGHT 1 & STANOLND OIL CO & 7030 & 0.56 & -102.64819034 & 31.26486416 & & 1.3 & 12119 & $-9,728$ \\
\hline 51 & WA WRIGHT 1 & STANOLND OIL CO & 9130 & 0.57 & -102.64819034 & 31.26486416 & & 2 & 13660 & $-11,269$ \\
\hline
\end{tabular}




\begin{tabular}{|c|c|c|c|c|c|c|c|c|c|c|}
\hline Map ID & Lease Name & Operator & Depth (ft) & \% Ro & Longitude & Latitude & Elev & \% Ro & Depth (ft) & Depth (-SL) \\
\hline 51 & WA WRIGHT 1 & STANOLND OIL CO & 8620 & 0.61 & -102.64819034 & 31.26486416 & & & & \\
\hline 51 & WA WRIGHT 1 & STANOLND OIL CO & 10035 & 0.66 & -102.64819034 & 31.26486416 & & & & \\
\hline 51 & WA WRIGHT 1 & STANOLND OIL CO & 11030 & 0.75 & -102.64819034 & 31.26486416 & & & & \\
\hline 51 & WA WRIGHT 1 & STANOLND OIL CO & 9630 & 0.79 & -102.64819034 & 31.26486416 & & & & \\
\hline 52 & ALAMO 1 & PHILLIPS PET ET AL & 8520 & 0.80 & -102.93526663 & 30.74683586 & 3562 & 0.6 & 7045 & $-3,483$ \\
\hline 52 & ALAMO 1 & PHILLIPS PET ET AL & 11725 & 0.95 & -102.93526663 & 30.74683586 & & 1.3 & 13482 & $-9,920$ \\
\hline 52 & ALAMO 1 & PHILLIPS PET ET AL & 13325 & 1.22 & -102.93526663 & 30.74683586 & & 2 & 17069 & $-13,507$ \\
\hline 52 & ALAMO 1 & PHILLIPS PET ET AL & 15030 & 1.55 & -102.93526663 & 30.74683586 & & & & \\
\hline 52 & ALAMO 1 & PHILLIPS PET ET AL & 16720 & 2.01 & -102.93526663 & 30.74683586 & & & & \\
\hline 53 & CROCKETT 1 & PENNZOIL CO INC & 8040 & 0.76 & -102.92657202 & 31.24062469 & 2533 & 0.6 & 6464 & $-3,931$ \\
\hline 53 & CROCKETT 1 & PENNZOIL CO INC & 7040 & 0.85 & -102.92657202 & 31.24062469 & & 1.3 & 11065 & $-8,532$ \\
\hline 53 & CROCKETT 1 & PENNZOIL CO INC & 10915 & 0.93 & -102.92657202 & 31.24062469 & & 2 & 13628 & $-11,095$ \\
\hline 53 & CROCKETT 1 & PENNZOIL CO INC & 9715 & 1.13 & -102.92657202 & 31.24062469 & & & & \\
\hline 54 & STROMAN WA B 1 & HUMBLE OIL \& REFG & 11030 & 1.09 & -102.75518325 & 30.91760853 & 2948 & 0.6 & 4261 & $-1,313$ \\
\hline 54 & STROMAN WA B 1 & HUMBLE OIL \& REFG & 10130 & 1.10 & -102.75518325 & 30.91760853 & & 1.3 & 12037 & $-9,089$ \\
\hline 54 & STROMAN WA B 1 & HUMBLE OIL \& REFG & 12040 & 1.23 & -102.75518325 & 30.91760853 & & 2 & 16370 & $-13,422$ \\
\hline 54 & STROMAN WA B 1 & HUMBLE OIL \& REFG & 13040 & 1.68 & -102.75518325 & 30.91760853 & & & & \\
\hline 54 & STROMAN WA B 1 & HUMBLE OIL \& REFG & 14060 & 1.71 & -102.75518325 & 30.91760853 & & & & \\
\hline 54 & STROMAN WA B 1 & HUMBLE OIL \& REFG & 15040 & 1.83 & -102.75518325 & 30.91760853 & & & & \\
\hline 54 & STROMAN WA B 1 & HUMBLE OIL \& REFG & 17040 & 2.05 & -102.75518325 & 30.91760853 & & & & \\
\hline 54 & STROMAN WA B 1 & HUMBLE OIL \& REFG & 19040 & 2.34 & -102.75518325 & 30.91760853 & & & & \\
\hline 55 & J ALONICK LJ 3 & SUN OIL COMPANY & 6793 & 0.41 & -101.76026231 & 31.56056592 & 2692 & 0.6 & 11214 & $-8,522$ \\
\hline 55 & J ALONICK LJ 3 & SUN OIL COMPANY & 7720 & 0.42 & -101.76026231 & 31.56056592 & & 1.3 & 20191 & $-17,499$ \\
\hline 55 & JALONICK LJ 3 & SUN OIL COMPANY & 6964 & 0.42 & -101.76026231 & 31.56056592 & & 2 & 25193 & $-22,501$ \\
\hline 55 & J ALONICK LJ 3 & SUN OIL COMPANY & 6920 & 0.42 & -101.76026231 & 31.56056592 & & & & \\
\hline 55 & J ALONICK LJ 3 & SUN OIL COMPANY & 6884 & 0.42 & -101.76026231 & 31.56056592 & & & & \\
\hline 55 & JALONICK LJ 3 & SUN OI L COMPANY & 6876 & 0.42 & -101.76026231 & 31.56056592 & & & & \\
\hline 56 & TURNER PRICE ETAL 1 & TIDEWATER OIL CO & 5950 & 0.38 & -101.67764 & 31.33611 & & \multirow{6}{*}{\multicolumn{3}{|c|}{ Location not used in Figures 2-4 }} \\
\hline 56 & TURNER PRICE ETAL 1 & TIDEWATER OIL CO & 7040 & 0.51 & -101.67764 & 31.33611 & & & & \\
\hline 56 & TURNER PRICE ETAL 1 & TIDEWATER OIL CO & 8040 & 0.69 & -101.67764 & 31.33611 & & & & \\
\hline 56 & TURNER PRICE ETAL 1 & TIDEWATER OIL CO & 9040 & 1.15 & -101.67764 & 31.33611 & & & & \\
\hline 56 & TURNER PRICE ETAL 1 & TIDEWATER OIL CO & 10040 & 1.16 & -101.67764 & 31.33611 & & & & \\
\hline 56 & TURNER PRICE ETAL 1 & TIDEWATER OIL CO & 9540 & 1.24 & -101.67764 & 31.33611 & & & & \\
\hline 57 & MRS VL SHURTLEFF 1 & GULF OIL \& PHILLIPS & 5000 & 0.42 & -103.70668495 & 31.3996191 & 2823 & 0.6 & 6918 & $-4,095$ \\
\hline 57 & MRS VL SHURTLEFF 1 & GULF OIL \& PHILLIPS & 7050 & 0.54 & -103.70668495 & 31.3996191 & & 1.3 & 10314 & $-7,491$ \\
\hline 57 & MRS VL SHURTLEFF 1 & GULF OIL \& PHILLIPS & 6030 & 0.55 & -103.70668495 & 31.3996191 & & 2 & 12206 & $-9,383$ \\
\hline 57 & MRS VL SHURTLEFF 1 & GULF OIL \& PHILLIPS & 8120 & 0.76 & -103.70668495 & 31.3996191 & & & & \\
\hline 57 & MRS VL SHURTLEFF 1 & GULF OIL \& PHILLIPS & 9100 & 1.01 & -103.70668495 & 31.3996191 & & & & \\
\hline 57 & MRS VL SHURTLEFF 1 & GULF OIL \& PHILLIPS & 10050 & 1.05 & -103.70668495 & 31.3996191 & & & & \\
\hline 57 & MRS VL SHURTLEFF 1 & GULF OIL \& PHILLIPS & 10840 & 1.63 & -103.70668495 & 31.3996191 & & & & \\
\hline
\end{tabular}




\begin{tabular}{|c|c|c|c|c|c|c|c|c|c|c|}
\hline Map ID & Lease Name & Operator & Depth (ft) & $\%$ Ro & Longitude & Latitude & Elev & \% Ro & Depth (ft) & Depth (-SL) \\
\hline 58 & WAPLES-PLATTER 1 & HAMON J AKE L & 7520 & 0.45 & -103.3158729 & 31.12864431 & 2814 & 0.6 & 9289 & $-6,475$ \\
\hline 58 & WAPLES-PLATTER 1 & HAMON J AKE L & 9020 & 0.46 & -103.3158729 & 31.12864431 & & 1.3 & 14097 & $-11,283$ \\
\hline 58 & WAPLES-PLATTER 1 & HAMON J AKE L & 10515 & 0.61 & -103.3158729 & 31.12864431 & & 2 & 16776 & $-13,962$ \\
\hline 58 & WAPLES-PLATTER 1 & HAMON J AKE L & 13570 & 1.14 & -103.3158729 & 31.12864431 & & & & \\
\hline 58 & WAPLES-PLATTER 1 & HAMON J AKE L & 6110 & 1.26 & -103.3158729 & 31.12864431 & & & & \\
\hline 58 & WAPLES-PLATTER 1 & HAMON J AKE L & 15015 & 1.52 & -103.3158729 & 31.12864431 & & & & \\
\hline 58 & WAPLES-PLATTER 1 & HAMON J AKE L & 16515 & 1.81 & -103.3158729 & 31.12864431 & & & & \\
\hline 58 & WAPLES-PLATTER 1 & HAMON J AKE L & 21420 & 1.99 & -103.3158729 & 31.12864431 & & & & \\
\hline 59 & HORRY L ETAL 3 & GULF OIL CORP & 7020 & 0.38 & -103.25995211 & 31.35712398 & 2598 & 0.6 & 8953 & $-6,355$ \\
\hline 59 & HORRY L ETAL 3 & GULF OIL CORP & 11320 & 1.17 & -103.25995211 & 31.35712398 & & 1.3 & 12335 & $-9,737$ \\
\hline 59 & HORRY L ETAL 3 & GULF OIL CORP & 13020 & 1.36 & -103.25995211 & 31.35712398 & & 2 & 14219 & $-11,621$ \\
\hline 59 & HORRY L ETAL 3 & GULF OIL CORP & 13020 & 1.36 & -103.25995211 & 31.35712398 & & & & \\
\hline 60 & TENNEY GERALD E 1 & PAN AMERICAN & 6040 & 0.44 & -103.57740649 & 30.92966809 & 3066 & 0.6 & 7422 & $-4,356$ \\
\hline 60 & TENNEY GERALD E 1 & PAN AMERICAN & 7005 & 0.57 & -103.57740649 & 30.92966809 & & 1.3 & 11309 & $-8,243$ \\
\hline 60 & TENNEY GERALD E 1 & PAN AMERICAN & 8040 & 0.70 & -103.57740649 & 30.92966809 & & 2 & 13474 & $-10,408$ \\
\hline 60 & TENNEY GERALD E 1 & PAN AMERICAN & 9040 & 0.84 & -103.57740649 & 30.92966809 & & & & \\
\hline 60 & TENNEY GERALD E 1 & PAN AMERI CAN & 11040 & 1.08 & -103.57740649 & 30.92966809 & & & & \\
\hline 60 & TENNEY GERALD E 1 & PAN AMERICAN & 10240 & 1.15 & -103.57740649 & 30.92966809 & & & & \\
\hline 61 & JS GOLDEN-SUN 1 & LOWE RALPH L & 4030 & 0.50 & -101.01822971 & 32.64456582 & 2340 & 0.6 & 4795 & $-2,455$ \\
\hline 61 & JS GOLDEN-SUN 1 & LOWE RALPH L & 3530 & 0.51 & -101.01822971 & 32.64456582 & & 1.3 & 9671 & $-7,331$ \\
\hline 61 & JS GOLDEN-SUN 1 & LOWE RALPH L & 4970 & 0.64 & -101.01822971 & 32.64456582 & & 2 & 12388 & $-10,048$ \\
\hline 61 & JS GOLDEN-SUN 1 & LOWE RALPH L & 6030 & 0.77 & -101.01822971 & 32.64456582 & & & & \\
\hline 61 & JS GOLDEN-SUN 1 & LOWE RALPH L & 7570 & 0.87 & -101.01822971 & 32.64456582 & & & & \\
\hline 61 & JS GOLDEN-SUN 1 & LOWE RALPH L & 6930 & 0.91 & -101.01822971 & 32.64456582 & & & & \\
\hline 62 & FG KEEL 1 & SOHIO PETROLEUM & 9130 & 0.42 & -101.91000 & 31.52000 & & & & \\
\hline 62 & FG KEEL 1 & SOHIO PETROLEUM & 6050 & 0.45 & -101.91078 & 31.51920 & & \multicolumn{3}{|c|}{ Location not used in Figures 2-4 } \\
\hline 62 & FG KEEL 1 & SOHIO PETROLEUM & 11455 & 0.46 & -101.91078 & 31.51920 & & & & \\
\hline 62 & FG KEEL 1 & SOHIO PETROLEUM & 9970 & 0.53 & -101.91078 & 31.51920 & & & & \\
\hline 62 & FG KEEL 1 & SOHIO PETROLEUM & 8150 & 0.54 & -101.91078 & 31.51920 & & & & \\
\hline 62 & FG KEEL 1 & SOHIO PETROLEUM & 7040 & 0.58 & -101.91078 & 31.51920 & & & & \\
\hline 63 & KEEL FG 1 & HUMBLE OIL \& REFG CO & 4530 & 0.45 & -101.91000 & 31.51000 & & & & \\
\hline 63 & KEEL FG 1 & HUMBLE OIL \& REFG CO & 3465 & 0.49 & -101.91000 & 31.51000 & & \multicolumn{3}{|c|}{ Location not used in Figures 2-4 } \\
\hline 63 & KEEL FG 1 & HUMBLE OIL \& REFG CO & 5550 & 0.83 & -101.91000 & 31.51000 & & & & \\
\hline 63 & KEEL FG 1 & HUMBLE OIL \& REFG CO & 7005 & 1.08 & -101.91000 & 31.51000 & & & & \\
\hline 64 & CAMPBELL FLORENCE 1 & LEVIN M - PATTON R & 6325 & 0.52 & -102.10297 & 31.24538607 & 2735 & 0.6 & 7632 & $-4,897$ \\
\hline 64 & CAMPBELL FLORENCE 1 & LEVIN M - PATTON R & 8430 & 0.57 & -102.10296903 & 31.24538607 & & 1.3 & 10317 & $-7,582$ \\
\hline 64 & CAMPBELL FLORENCE 1 & LEVIN M - PATTON R & 9430 & 0.94 & -102.10296903 & 31.24538607 & & 2 & 11813 & $-9,078$ \\
\hline 64 & CAMPBELL FLORENCE 1 & LEVIN M - PATTON R & 7325 & 1.03 & -102.10296903 & 31.24538607 & & & & \\
\hline 64 & CAMPBELL FLORENCE 1 & LEVIN M - PATTON R & 10425 & 1.10 & -102.10296903 & 31.24538607 & & & & \\
\hline
\end{tabular}




\begin{tabular}{|c|c|c|c|c|c|c|c|c|c|c|}
\hline Map ID & Lease Name & Operator & Depth (ft) & $\%$ Ro & Longitude & Latitude & Elev & $\%$ Ro & Depth (ft) & Depth (-SL) \\
\hline 65 & J OHN EDWARDS ETAL E 1 & GULF OIL CORP & 7305 & 0.60 & -102.84596732 & 31.59347608 & 2662 & 0.6 & 7547 & $-4,885$ \\
\hline 65 & J OHN EDWARDS ETAL E 1 & GULF OIL CORP & 9070 & 0.79 & -102.84596732 & 31.59347608 & & 1.3 & 9684 & $-7,022$ \\
\hline 65 & J OHN EDWARDS ETAL E 1 & GULF OIL CORP & 8230 & 1.03 & -102.84596732 & 31.59347608 & & 2 & 10874 & $-8,212$ \\
\hline 65 & J OHN EDWARDS ETAL E 1 & GULF OIL CORP & 10015 & 1.32 & -102.84596732 & 31.59347608 & & & & \\
\hline 66 & GREER-MCGI NLEAS UNT 1 & GULF OIL CORP & 8530 & 0.37 & -103.41132883 & 31.61537986 & 2693 & 0.6 & 9772 & $-7,079$ \\
\hline 66 & GREER-MCGI NLEAS UNT 1 & GULF OIL CORP & 9030 & 0.58 & -103.41132883 & 31.61537986 & & 1.3 & 14120 & $-11,427$ \\
\hline 66 & GREER-MCGI NLEAS UNT 1 & GULF OIL CORP & 10040 & 0.68 & -103.41132883 & 31.61537986 & & 2 & 16543 & $-13,850$ \\
\hline 66 & GREER-MCGI NLEAS UNT 1 & GULF OIL CORP & 14030 & 1.65 & -103.41132883 & 31.61537986 & & & & \\
\hline 66 & GREER-MCGI NLEAS UNT 1 & GULF OIL CORP & 15030 & 1.93 & -103.41132883 & 31.61537986 & & & & \\
\hline 66 & GREER-MCGI NLEAS UNT 1 & GULF OIL CORP & 16040 & 2.20 & -103.41132883 & 31.61537986 & & & & \\
\hline 66 & GREER-MCGI NLEAS UNT 1 & GULF OIL CORP & 17050 & 2.27 & -103.41132883 & 31.61537986 & & & & \\
\hline 66 & GREER-MCGI NLEAS UNT 1 & GULF OIL CORP & 19540 & 2.71 & -103.41132883 & 31.61537986 & & & & \\
\hline 66 & GREER-MCGI NLEAS UNT 1 & GULF OIL CORP & 20360 & 2.92 & -103.41132883 & 31.61537986 & & & & \\
\hline 67 & UNIVERSITY PYOTE UN 1 & UNION OIL OF CAL & 5520 & 0.44 & -103.16592204 & 31.54442445 & 2635 & 0.6 & 8445 & $-5,810$ \\
\hline 67 & UNI VERSITY PYOTE UN 1 & UNION OIL OF CAL & 7550 & 0.47 & -103.16592204 & 31.54442445 & & 1.3 & 12305 & $-9,670$ \\
\hline 67 & UNIVERSITY PYOTE UN 1 & UNION OIL OF CAL & 6550 & 0.49 & -103.16592204 & 31.54442445 & & 2 & 14455 & $-11,820$ \\
\hline 67 & UNIVERSITY PYOTE UN 1 & UNION OIL OF CAL & 11530 & 0.69 & -103.16592204 & 31.54442445 & & & & \\
\hline 67 & UNIVERSITY PYOTE UN 1 & UNION OIL OF CAL & 12520 & 0.82 & -103.16592204 & 31.54442445 & & & & \\
\hline 67 & UNIVERSITY PYOTE UN 1 & UNION OIL OF CAL & 10530 & 0.86 & -103.16592204 & 31.54442445 & & & & \\
\hline 67 & UNIVERSITY PYOTE UN 1 & UNION OIL OF CAL & 8540 & 0.86 & -103.16592204 & 31.54442445 & & & & \\
\hline 67 & UNIVERSITY PYOTE UN 1 & UNION OIL OF CAL & 9530 & 1.09 & -103.16592204 & 31.54442445 & & & & \\
\hline 67 & UNIVERSITY PYOTE UN 1 & UNION OIL OF CAL & 12430 & 1.15 & -103.16592204 & 31.54442445 & & & & \\
\hline 67 & UNIVERSITY PYOTE UN 1 & UNION OIL OF CAL & 14235 & 2.07 & -103.16592204 & 31.54442445 & & & & \\
\hline 68 & ROARK IC 1 & PAN AMERICAN & 5620 & 0.42 & -103.18313378 & 31.83313079 & 2840 & 0.6 & 7763 & $-4,923$ \\
\hline 68 & ROARK IC 1 & PAN AMERICAN & 6530 & 0.59 & -103.18313378 & 31.83313079 & & 1.3 & 12523 & $-9,683$ \\
\hline 68 & ROARK IC 1 & PAN AMERICAN & 11030 & 0.66 & -103.18313378 & 31.83313079 & & 2 & 15176 & $-12,336$ \\
\hline 68 & ROARK IC 1 & PAN AMERICAN & 9240 & 0.85 & -103.18313378 & 31.83313079 & & & & \\
\hline 68 & ROARK IC 1 & PAN AMERICAN & 7645 & 0.86 & -103.18313378 & 31.83313079 & & & & \\
\hline 68 & ROARK IC 1 & PAN AMERICAN & 12945 & 1.04 & -103.18313378 & 31.83313079 & & & & \\
\hline 68 & ROARK IC 1 & PAN AMERICAN & 10540 & 1.15 & -103.18313378 & 31.83313079 & & & & \\
\hline 68 & ROARK IC 1 & PAN AMERICAN & 13430 & 1.29 & -103.18313378 & 31.83313079 & & & & \\
\hline 68 & ROARK IC 1 & PAN AMERICAN & 12630 & 1.35 & -103.18313378 & 31.83313079 & & & & \\
\hline 69 & Humble $1 \mathrm{CV}$ University & Humble & 5810 & 0.76 & -101.48509331 & 30.64708246 & 2367 & 0.6 & 7371 & $-5,004$ \\
\hline 69 & Humble $1 \mathrm{CV}$ University & Humble & 6210 & 0.74 & -101.48509331 & 30.64708246 & & 1.3 & 8409 & $-6,042$ \\
\hline 69 & Humble 1CV University & Humble & 6510 & 0.94 & -101.48509331 & 30.64708246 & & 2 & 8987 & $-6,620$ \\
\hline 69 & Humble $1 \mathrm{CV}$ University & Humble & 6810 & 1.17 & -101.48509331 & 30.64708246 & & & & \\
\hline 69 & Humble $1 \mathrm{CV}$ University & Humble & 7310 & 1.09 & -101.48509331 & 30.64708246 & & & & \\
\hline 69 & Humble $1 \mathrm{CV}$ University & Humble & 7710 & 1.35 & -101.48509331 & 30.64708246 & & & & \\
\hline 69 & Humble $1 \mathrm{CV}$ University & Humble & 8210 & 1.19 & -101.48509331 & 30.64708246 & & & & \\
\hline 69 & Humble 1 CV University & Humble & 8610 & 0.71 & -101.48509331 & 30.64708246 & & & & \\
\hline 69 & Humble $1 \mathrm{CV}$ University & Humble & 9110 & 0.80 & -101.48509331 & 30.64708246 & & & & \\
\hline
\end{tabular}




\begin{tabular}{|c|c|c|c|c|c|c|c|c|c|c|}
\hline Map ID & Lease Name & Operator & Depth (ft) & $\%$ Ro & Longitude & Latitude & Elev & $\%$ Ro & Depth (ft) & Depth (-SL) \\
\hline 69 & Humble 1CV University & Humble & 9610 & 0.93 & -101.48509331 & 30.64708246 & & & & \\
\hline 69 & Humble 1CV University & Humble & 10005 & 0.99 & -101.48509331 & 30.64708246 & & & & \\
\hline 69 & Humble 1CV University & Humble & 10210 & 1.16 & -101.48509331 & 30.64708246 & & & & \\
\hline 70 & \#1 Ellis Leah Marie & Tarpon & 4010 & 0.79 & -100.92035558 & 31.20258233 & 2486 & 0.6 & 3769 & $-1,283$ \\
\hline 70 & \#1 Ellis Leah Marie & Tarpon & 5810 & 0.77 & -100.92035558 & 31.20258233 & & 1.3 & 8597 & $-6,111$ \\
\hline 70 & \#1 Ellis Leah Marie & Tarpon & 6310 & 0.93 & -100.92035558 & 31.20258233 & & 2 & 11288 & $-8,802$ \\
\hline 70 & \#1 Ellis Leah Marie & Tarpon & 6910 & 0.82 & -100.92035558 & 31.20258233 & & & & \\
\hline 71 & \#1 H.E. Fisher & Plymouth & 3040 & 0.33 & -101.18140109 & 33.42411529 & 2490 & 0.6 & 6040 & $-3,550$ \\
\hline 71 & \#1 H.E. Fisher & Plymouth & 3530 & 0.29 & -101.18140109 & 33.42411529 & & 1.3 & 8569 & $-6,079$ \\
\hline 71 & \#1 H.E. Fisher & Plymouth & 5640 & 0.57 & -101.18140109 & 33.42411529 & & 2 & 9978 & $-7,488$ \\
\hline 71 & \#1 H.E. Fisher & Plymouth & 6140 & 1.05 & -101.18140109 & 33.42411529 & & & & \\
\hline 71 & \#1 H.E. Fisher & Plymouth & 6775 & 0.66 & -101.18140109 & 33.42411529 & & & & \\
\hline 71 & \#1 H.E. Fisher & Plymouth & 7250 & 0.55 & -101.18140109 & 33.42411529 & & & & \\
\hline 71 & \#1 H.E. Fisher & Plymouth & 7940 & 0.76 & -101.18140109 & 33.42411529 & & & & \\
\hline 71 & \#1 H.E. Fisher & Plymouth & 8260 & 1.15 & -101.18140109 & 33.42411529 & & & & \\
\hline 72 & Big Lake & University D 1 & 4810 & 0.98 & -101.70332801 & 31.23761533 & 2683 & 0.6 & 5655 & $-2,972$ \\
\hline 72 & Big Lake & University D 1 & 5510 & 0.78 & -101.70332801 & 31.23761533 & & 1.3 & 8591 & $-5,908$ \\
\hline 72 & Big Lake & University D 1 & 5910 & 0.74 & -101.70332801 & 31.23761533 & & 2 & 10227 & $-7,544$ \\
\hline 72 & Big Lake & University D 1 & 6425 & 0.93 & -101.70332801 & 31.23761533 & & & & \\
\hline 72 & Big Lake & University D 1 & 6807 & 0.96 & -101.70332801 & 31.23761533 & & & & \\
\hline 72 & Big Lake & University D 1 & 7805 & 1.27 & -101.70332801 & 31.23761533 & & & & \\
\hline 72 & Big Lake & University D 1 & 8255 & 1.02 & -101.70332801 & 31.23761533 & & & & \\
\hline 72 & Big Lake & University D 1 & 8803 & 0.77 & -101.70332801 & 31.23761533 & & & & \\
\hline 72 & Big Lake & University D 1 & 9355 & 0.91 & -101.70332801 & 31.23761533 & & & & \\
\hline 72 & Big Lake & University D 1 & 9905 & 1.04 & -101.70332801 & 31.23761533 & & & & \\
\hline 73 & WD \#1 Wilson & Hamon Jake L & 11410 & 1.18 & -103.29834792 & 31.12254438 & 2796 & 0.6 & 8892 & $-6,096$ \\
\hline 73 & WD \#1 Wilson & Hamon J ake L & 12510 & 1.24 & -103.29834792 & 31.12254438 & & 1.3 & 14366 & $-11,570$ \\
\hline 73 & WD \#1 Wilson & Hamon Jake L & 13310 & 1.09 & -103.29834792 & 31.12254438 & & 2 & 17416 & $-14,620$ \\
\hline 73 & WD \#1 Wilson & Hamon J ake L & 12150 & 1.10 & -103.29834792 & 31.12254438 & & & & \\
\hline 73 & WD \#1 Wilson & Hamon J ake L & 14110 & 1.38 & -103.29834792 & 31.12254438 & & & & \\
\hline 73 & WD \#1 Wilson & Hamon J ake L & 14810 & 1.39 & -103.29834792 & 31.12254438 & & & & \\
\hline 73 & WD \#1 Wilson & Hamon Jake L & 15525 & 1.57 & -103.29834792 & 31.12254438 & & & & \\
\hline 73 & WD \#1 Wilson & Hamon J ake L & 16615 & 1.53 & -103.29834792 & 31.12254438 & & & & \\
\hline 73 & WD \#1 Wilson & Hamon J ake L & 16815 & 1.07 & -103.29834792 & 31.12254438 & & & & \\
\hline 73 & WD \#1 Wilson & Hamon J ake L & 17465 & 1.85 & -103.29834792 & 31.12254438 & & & & \\
\hline 74 & \#6 Mitchell & Shell & 1710 & 0.67 & -101.59128134 & 30.35157892 & 2286 & 0.6 & 69 & 2,217 \\
\hline 74 & \#6 Mitchell & Shell & 4410 & 0.99 & -101.59128134 & 30.35157892 & & 1.3 & 6709 & $-4,423$ \\
\hline 74 & \#6 Mitchell & Shell & 5210 & 1.09 & -101.59128134 & 30.35157892 & & 2 & 10408 & $-8,122$ \\
\hline 74 & \#6 Mitchell & Shell & 5910 & 1.33 & -101.59128134 & 30.35157892 & & & & \\
\hline 74 & \#6 Mitchell & Shell & 6610 & 1.45 & -101.59128134 & 30.35157892 & & & & \\
\hline 74 & \#6 Mitchell & Shell & 7310 & 1.38 & -101.59128134 & 30.35157892 & & & & \\
\hline
\end{tabular}




\begin{tabular}{|c|c|c|c|c|c|c|c|c|c|c|}
\hline Map ID & Lease Name & Operator & Depth (ft) & \% Ro & Longitude & Latitude & Elev & \% Ro & Depth (ft) & Depth (-SL) \\
\hline 74 & \#6 Mitchell & Shell & 8010 & 1.53 & -101.59128134 & 30.35157892 & & & & \\
\hline 74 & \#6 Mitchell & Shell & 8710 & 1.64 & -101.59128134 & 30.35157892 & & & & \\
\hline 74 & \#6 Mitchell & Shell & 9410 & 1.80 & -101.59128134 & 30.35157892 & & & & \\
\hline 74 & \#6 Mitchell & Shell & 10010 & 1.84 & -101.59128134 & 30.35157892 & & & & \\
\hline 74 & \#6 Mitchell & Shell & 10710 & 2.10 & -101.59128134 & 30.35157892 & & & & \\
\hline 74 & \#6 Mitchell & Shell & 12210 & 2.15 & -101.59128134 & 30.35157892 & & & & \\
\hline 75 & 1 W. Massie \#28 & Atlantic & 4910 & 1.43 & -101.20957556 & 30.2905338 & 2159 & 0.6 & 436 & 1,723 \\
\hline 75 & 1 W. Massie \#28 & Atlantic & 5710 & 1.63 & -101.20957556 & 30.2905338 & & 1.3 & 4937 & $-2,778$ \\
\hline 75 & 1 W. Massie \#28 & Atlantic & 6110 & 1.71 & -101.20957556 & 30.2905338 & & 2 & 7445 & $-5,286$ \\
\hline 75 & 1 W. Massie \#28 & Atlantic & 6810 & 1.8 & -101.20957556 & 30.2905338 & & & & \\
\hline 75 & 1W. Massie \#28 & Atlantic & 7410 & 2.03 & -101.20957556 & 30.2905338 & & & & \\
\hline 75 & 1 W. Massie \#28 & Atlantic & 8010 & 2.2 & -101.20957556 & 30.2905338 & & & & \\
\hline 75 & $1 \mathrm{~W}$. Massie \#28 & Atlantic & 8610 & 2.39 & -101.20957556 & 30.2905338 & & & & \\
\hline 75 & 1 W. Massie \#28 & Atlantic & 9110 & 2.29 & -101.20957556 & 30.2905338 & & & & \\
\hline 75 & 1 W. Massie \#28 & Atlantic & 9710 & 2.73 & -101.20957556 & 30.2905338 & & & & \\
\hline 75 & 1 W. Massie \#28 & Atlantic & 10510 & 2.97 & -101.20957556 & 30.2905338 & & & & \\
\hline 75 & 1 W. Massie \#28 & Atlantic & 11440 & 3.36 & -101.20957556 & 30.2905338 & & & & \\
\hline 75 & 1 W. Massie \#28 & Atlantic & 11560 & 5.26 & -101.20957556 & 30.2905338 & & & & \\
\hline 76 & Sugg Ela C. A. & Cactus Drilling & 5110 & 1.19 & -101.10571224 & 31.31732383 & 2507 & 0.6 & 3395 & -888 \\
\hline 76 & Sugg Ela C. A. & Cactus Drilling & 5710 & 0.92 & -101.10571224 & 31.31732383 & & 1.3 & 7812 & $-5,305$ \\
\hline 76 & Sugg Ela C. A. & Cactus Drilling & 6310 & 1 & -101.10571224 & 31.31732383 & & 2 & 10273 & $-7,766$ \\
\hline 76 & Sugg Ela C. A. & Cactus Drilling & 6810 & 1.05 & -101.10571224 & 31.31732383 & & & & \\
\hline 76 & Sugg Ela C. A. & Cactus Drilling & 7305 & 1.31 & -101.10571224 & 31.31732383 & & & & \\
\hline 76 & Sugg Ela C. A. & Cactus Drilling & 7805 & 1.07 & -101.10571224 & 31.31732383 & & & & \\
\hline 76 & Sugg Ela C. A. & Cactus Drilling & 8595 & 1.4 & -101.10571224 & 31.31732383 & & & & \\
\hline 76 & Sugg Ela C. A. & Cactus Drilling & 9010 & 1.3 & -101.10571224 & 31.31732383 & & & & \\
\hline 77 & \#1 Miller & Consolidated O \& G & 4995 & 0.5 & -101.24821493 & 32.98803225 & 2384 & 0.6 & 5869 & $-3,485$ \\
\hline 77 & \#1 Miller & Consolidated $\mathrm{O} \& \mathrm{G}$ & 6150 & 0.6 & -101.24821493 & 32.98803225 & & 1.3 & 8377 & $-5,993$ \\
\hline 77 & \#1 Miller & Consolidated $\mathrm{O} \& \mathrm{G}$ & 5310 & 0.49 & -101.24821493 & 32.98803225 & & 2 & 9775 & $-7,391$ \\
\hline 77 & \#1 Miller & Consolidated O \& G & 6710 & 0.96 & -101.24821493 & 32.98803225 & & & & \\
\hline 77 & \#1 Miller & Consolidated $\mathrm{O} \& \mathrm{G}$ & 7560 & 0.93 & -101.24821493 & 32.98803225 & & & & \\
\hline 77 & \#1 Miller & Consolidated O \& G & 7940 & 1.03 & -101.24821493 & 32.98803225 & & & & \\
\hline 78 & \#1 Mid Seale & Humble O \& R & 5410 & 0.45 & -101.45514809 & 33.34648065 & 2215 & 0.6 & 6224 & $-4,009$ \\
\hline 78 & \#1 Mid Seale & Humble $O \& R$ & 6010 & 1.01 & -101.45514809 & 33.34648065 & & 1.3 & 8167 & $-5,952$ \\
\hline 78 & \#1 Mid Seale & Humble $O \& R$ & 6910 & 1.11 & -101.45514809 & 33.34648065 & & 2 & 9250 & $-7,035$ \\
\hline 78 & \#1 Mid Seale & Humble $O \& R$ & 7410 & 0.88 & -101.45514809 & 33.34648065 & & & & \\
\hline 78 & \#1 Mid Seale & Humble $O \& R$ & 8010 & 0.99 & -101.45514809 & 33.34648065 & & & & \\
\hline 78 & \#1 Mid Seale & Humble $O \& R$ & 8420 & 1.05 & -101.45514809 & 33.34648065 & & & & \\
\hline 78 & \#1 Mid Seale & Humble $O \& R$ & 8515 & 1.03 & -101.45514809 & 33.34648065 & & & & \\
\hline 79 & \#47 Elsinore Royalty Co & Hunt Oil Co & 6990 & 0.9 & -103.11061392 & 30.62679122 & 3742 & 0.6 & 4407 & -665 \\
\hline 79 & \#47 Elsinore Royalty Co & Hunt Oil Co & 8750 & 0.97 & -103.11061392 & 30.62679122 & & 1.3 & 11116 & $-7,374$ \\
\hline
\end{tabular}




\begin{tabular}{|c|c|c|c|c|c|c|c|c|c|c|}
\hline Map ID & Lease Name & Operator & Depth (ft) & \% Ro & Longitude & Latitude & Elev & \% Ro & Depth (ft) & Depth (-SL) \\
\hline 79 & \#47 Elsinore Royalty Co & Hunt Oil Co & 9370 & 1 & -103.11061392 & 30.62679122 & & 2 & 14854 & $-11,112$ \\
\hline 79 & \#47 Elsinore Royalty Co & Hunt Oil Co & 10020 & 1.25 & -103.11061392 & 30.62679122 & & & & \\
\hline 79 & \#47 Elsinore Royalty Co & Hunt Oil Co & 10580 & 1.19 & -103.11061392 & 30.62679122 & & & & \\
\hline 79 & \#47 Elsinore Royalty Co & Hunt Oil Co & 11510 & 1.36 & -103.11061392 & 30.62679122 & & & & \\
\hline 79 & \#47 Elsinore Royalty Co & Hunt Oil Co & 12070 & 1.39 & -103.11061392 & 30.62679122 & & & & \\
\hline 79 & \#47 Elsinore Royalty Co & Hunt Oil Co & 12810 & 1.94 & -103.11061392 & 30.62679122 & & & & \\
\hline 79 & \#47 Elsinore Royalty Co & Hunt Oil Co & 14400 & 1.57 & -103.11061392 & 30.62679122 & & & & \\
\hline 79 & \#47 Elsinore Royalty Co & Hunt Oil Co & 15275 & 1.98 & -103.11061392 & 30.62679122 & & & & \\
\hline 80 & Pembrook 1-10 & McFarland Corp & 4610 & 0.55 & -101.46498235 & 31.22785011 & 2722 & 0.6 & 5682 & $-2,960$ \\
\hline 80 & Pembrook 1-11 & McFarland Corp & 4910 & 0.72 & -101.46498235 & 31.22785011 & & 1.3 & 9601 & $-6,879$ \\
\hline 80 & Pembrook 1-12 & McFarland Corp & 5710 & 0.68 & -101.46498235 & 31.22785011 & & 2 & 11784 & $-9,062$ \\
\hline 80 & Pembrook 1-13 & McFarland Corp & 6410 & 0.69 & -101.46498235 & 31.22785011 & & & & \\
\hline 80 & Pembrook 1-14 & McFarland Corp & 6905 & 1.02 & -101.46498235 & 31.22785011 & & & & \\
\hline 80 & Pembrook 1-15 & McFarland Corp & 7510 & 0.61 & -101.46498235 & 31.22785011 & & & & \\
\hline 80 & Pembrook 1-16 & McFarland Corp & 8110 & 1.18 & -101.46498235 & 31.22785011 & & & & \\
\hline 80 & Pembrook 1-17 & McFarland Corp & 8710 & 1.17 & -101.46498235 & 31.22785011 & & & & \\
\hline 80 & Pembrook 1-18 & McFarland Corp & 9305 & 1.02 & -101.46498235 & 31.22785011 & & & & \\
\hline 80 & Pembrook 1-19 & McFarland Corp & 9805 & 1.2 & -101.46498235 & 31.22785011 & & & & \\
\hline 80 & Pembrook 1-20 & McFarland Corp & 10350 & 0.93 & -101.46498235 & 31.22785011 & & & & \\
\hline 81 & Calvert A \#1 & Sinclair $O \&$ \& & 8610 & 1.03 & -103.06242 & 31.23932856 & 2610 & 0.6 & 6564 & $-3,954$ \\
\hline 81 & Calvert A \#2 & Sinclair $O \& G$ & 10210 & 1.02 & -103.06242 & 31.23932856 & & 1.3 & 11907 & $-9,297$ \\
\hline 81 & Calvert A \#3 & Sinclair $O \& G$ & 11110 & 1.05 & -103.06242 & 31.23932856 & & 2 & 14884 & $-12,274$ \\
\hline 81 & Calvert A \#4 & Sinclair $O \& G$ & 11710 & 1.11 & -103.06242 & 31.23932856 & & & & \\
\hline 81 & Calvert A \#5 & Sinclair $O \& G$ & 12220 & 1.23 & -103.06242 & 31.23932856 & & & & \\
\hline 81 & Calvert A \#6 & Sinclair $O \& G$ & 12240 & 1.48 & -103.06242 & 31.23932856 & & & & \\
\hline 81 & Calvert A \#7 & Sinclair $O \& G$ & 12420 & 1.4 & -103.06242 & 31.23932856 & & & & \\
\hline 82 & Lago Unit \#1 & Texaco Inc & 7910 & 0.75 & -103.85091809 & 31.91878197 & 2849 & 0.6 & 6837 & $-3,988$ \\
\hline 82 & Lago Unit \#2 & Texaco Inc & 8610 & 0.7 & -103.85091809 & 31.91878197 & & 1.3 & 11055 & $-8,206$ \\
\hline 82 & Lago Unit \#3 & Texaco Inc & 9210 & 0.86 & -103.85091809 & 31.91878197 & & 2 & 13404 & $-10,555$ \\
\hline 82 & Lago Unit \#4 & Texaco Inc & 9910 & 1.41 & -103.85091809 & 31.91878197 & & & & \\
\hline 82 & Lago Unit \#5 & Texaco Inc & 11210 & 1.45 & -103.85091809 & 31.91878197 & & & & \\
\hline 82 & Lago Unit \#6 & Texaco Inc & 11910 & 1.68 & -103.85091809 & 31.91878197 & & & & \\
\hline 82 & Lago Unit \#7 & Texaco Inc & 12410 & 1.78 & -103.85091809 & 31.91878197 & & & & \\
\hline 82 & Lago Unit \#8 & Texaco Inc & 12810 & 1.88 & -103.85091809 & 31.91878197 & & & & \\
\hline 82 & Lago Unit \#9 & Texaco Inc & 13310 & 2.22 & -103.85091809 & 31.91878197 & & & & \\
\hline 82 & Lago Unit \#10 & Texaco Inc & 14510 & 2.07 & -103.85091809 & 31.91878197 & & & & \\
\hline 82 & Lago Unit \#11 & Texaco Inc & 15210 & 2.25 & -103.85091809 & 31.91878197 & & & & \\
\hline 82 & Lago Unit \#12 & Texaco Inc & 16205 & 3.01 & -103.85091809 & 31.91878197 & & & & \\
\hline 83 & J ohnson W.D. \#1 & Sun Oil Co & 5435 & 0.7 & -103.56779628 & 31.73535291 & 2793 & 0.6 & 6425 & $-3,632$ \\
\hline 83 & Johnson W.D. \#1 & Sun Oil Co & 8450 & 0.55 & -103.56779628 & 31.73535291 & & 1.3 & 11546 & $-8,753$ \\
\hline 83 & Johnson W.D. \#1 & Sun Oil Co & 8710 & 0.73 & -103.56779628 & 31.73535291 & & 2 & 14399 & $-11,606$ \\
\hline 83 & Johnson W.D. \#1 & Sun Oil Co & 9075 & 1.25 & -103.56779628 & 31.73535291 & & & & \\
\hline
\end{tabular}




\begin{tabular}{|c|c|c|c|c|c|c|c|c|c|c|}
\hline Map ID & Lease Name & Operator & Depth (ft) & $\%$ Ro & Longitude & Latitude & Elev & $\%$ Ro & Depth (ft) & Depth (-SL) \\
\hline 83 & Johnson W.D. \#1 & Sun Oil Co & 9085 & 1.53 & -103.56779628 & 31.73535291 & & & & \\
\hline 83 & Johnson W.D. \#1 & Sun Oil Co & 9305 & 1.19 & -103.56779628 & 31.73535291 & & & & \\
\hline 83 & J ohnson W.D. \#1 & Sun Oil Co & 9825 & 0.93 & -103.56779628 & 31.73535291 & & & & \\
\hline 83 & Johnson W.D. \#1 & Sun Oil Co & 10065 & 0.89 & -103.56779628 & 31.73535291 & & & & \\
\hline 83 & J ohnson W.D. \#1 & Sun Oil Co & 11405 & 1.32 & -103.56779628 & 31.73535291 & & & & \\
\hline 83 & Johnson W.D. \#1 & Sun Oil Co & 11170 & 1 & -103.56779628 & 31.73535291 & & & & \\
\hline 83 & Johnson W.D. \#1 & Sun Oil Co & 12010 & 1.83 & -103.56779628 & 31.73535291 & & & & \\
\hline 83 & J ohnson W.D. \#1 & Sun Oil Co & 13410 & 1.68 & -103.56779628 & 31.73535291 & & & & \\
\hline 83 & Johnson W.D. \#1 & Sun Oil Co & 14410 & 1.75 & -103.56779628 & 31.73535291 & & & & \\
\hline 83 & Johnson W.D. \#1 & Sun Oil Co & 15420 & 1.84 & -103.56779628 & 31.73535291 & & & & \\
\hline 83 & Johnson W.D. \#1 & Sun Oil Co & 15865 & 1.9 & -103.56779628 & 31.73535291 & & & & \\
\hline 83 & J ohnson W.D. \#1 & Sun Oil Co & 16210 & 2.1 & -103.56779628 & 31.73535291 & & & & \\
\hline 83 & Johnson W.D. \#1 & Sun Oil Co & 17205 & 2.43 & -103.56779628 & 31.73535291 & & & & \\
\hline 84 & Owens B \#1 & Cities Senvice Oil & 3510 & 0.84 & -101.73000 & 30.79000 & & Locatio & not used in $\mathrm{Fi}$ & res 2-4 \\
\hline 84 & Owens B \#1 & Cities Service Oil & 4780 & 0.92 & -101.73000 & 30.79000 & & & & \\
\hline
\end{tabular}

